\title{
EVOLUCIÓN DE LAS CAUSAS Y EFECTOS DEL DESAJUSTE EDUCATIVO EN EL MERCADO LABORAL MEXICANO 1990-2010'
}

\author{
Héctor Alberto Botello Peñaloza ${ }^{2}$
}

Universidad Industrial de Santander - Artículo Tipo 1.

Investigación Científica y Tecnológica - Recibido 19 de Mayo 2014 - Aceptado: 13 de Junio 2015

doi: http://dx.doi.org/10.17981/econcuc.36.1.2015.30

\section{RESUMEN}

Este artículo estudió las causas y efectos del desajuste educativo en el mercado laboral mexicano, para lo cual se utilizaron los censos de población desde 1990 a 2010. Se encontró que el desajuste obedece a un problema estructural de la economía mexicana, que abarca en promedio el 31\% de la población ocupada, sin variaciones en los 20 años que fueron objeto de estudio. El desajuste educativo contribuye a explicar parte de las diferencias salariales con un rendimiento positivo para los trabajadores con sobre-educación y una penalización en el caso de la infra-educación. Se concluyó que las iniciativas de los observatorios laborales para egresados, para conocer las competencias sus competencias y las que necesitan los empresarios son un primer paso para solucionar el problema de sobre-educación en el país.

\section{Palabras Clave:}

Educación, Capital humano, Elección ocupacional, Productividad.

JEL: I21, I23, J01, J24

\section{Si va a referenciar este artículo}

Botello, M. (2015). Evolución de las causas y efectos del desajuste educativo en el mercado laboral mexicano 1990-2010. Económicas CUC, 36(1), 275-304.

doi: http://dx.doi.org/10.17981/econcuc.36.1.2015.30

\footnotetext{
${ }^{1}$ Artículo de investigación derivado del proyecto "Poblamientos y conflictos territoriales" del Grupo de Investigación Desarrollo Regional y Ordenamiento Territorial en la Línea de Investigación Desarrollo humano y conflictos, financiado por la Universidad Industrial de Santander.

${ }^{2}$ Catedrático Universidad Industrial de Santander, Bucaramanga. Colombia. Economista. Magister en Ingeniería Industrial. Correo: hectoralbertobotello@gmail.com.
} 


\section{INTRODUCCIÓN}

En las últimas décadas, los países han realizado importantes inversiones en educación, viéndose reflejado en el aumento de las tasas de escolaridad y en las tasas de participación en la educación terciaria. Sin embargo, parte de los conocimientos adquiridos a través de la educación pueden estar siendo infrautilizados si éstos no concuerdan con las competencias requeridas por las empresas. En este sentido, el desajuste educativo surge, cuando una persona se encuentra en un empleo que infravalora o sobrevalora la dotación de capital humano que posee (Freeman, 1975), existe evidencia de que tanto en países avanzados como en desarrollo, han crecido los diferentes tipos de desajustes educativos. (Mc Guiness, 2006)

La existencia de este fenómeno desestimula la inversión en educación, dado que las tasas de rendimiento esperado disminuyen (Mora, 2008). Diferentes explicaciones han sido planteadas para explicar este tipo de fenómenos, tales como fricciones temporales en el mercado laboral (Johnson, 1978; Sicherman \& Galor, 1990), información imperfecta (Jovanovic, 1979), inmovilidad geográfica (Frank, 1978), efectos de la señalización y movilidad laboral. (Rosen, 1972; Sicherman \& Galor, 1990; Sicherman, 1991)

De acuerdo con la teoría neoclásica, los cambios de población, llevan a un aumento desordenado del número de personas que invierten en educación, causando que las empresas no puedan cambiar rápidamente sus requisitos educativos, lo que puede provocar un desajuste estructural entre la oferta y la demanda laboral. La literatura empírica tiende a afirmar que los ingresos laborales devengados por los trabajadores, dependen de su capital humano acumulado como por las competencias desarrolladas dentro de la empresa, por lo tanto, si un trabajador esta sobre o infra educado tendrá una penalidad frente a los correctamente educados (Hartog, 2000). Desde otro punto de vista, los modelos de señalización plantean este fenómeno como un efecto permanente.

La educación es una señal utilizada por los trabajadores para mostrar en el mercado laboral que son más aptos y en consecuencia más productivos; de esta forma, los individuos invierten en educación para tener más oportunidades frente a otros trabajadores. En este sentido, puede suceder un incremento en la cantidad de personas con educación universitaria y por ende, saturar el mercado, causando un problema de sobre educación generalizada que no puede ser atendida por la estructura productiva del país.

No obstante, los desajustes también pueden ser positivos, dado que los individuos realizan temporalmente trabajos para los que están sobre-educados pues adquieren las habilidades necesarias para realizar trabajos más calificados; por esto se puede considerar que la movilidad ocupacional está relacionada con cierto grado de desajuste educativo. Con esto se puede llegar a decir que los desajustes solo serían un fenómeno temporal del mercado laboral y no un problema estructural entre la demanda y oferta de trabajo (Sicherman, 1991; Kiker, Santos \& De Oliveira, 1997). Para estudiar empíricamente los desajustes educativos, es necesario tener en cuenta la heterogeneidad de los trabajadores y las competencias realmente adquiridas por los mismos, ya que una parte del desajuste puede deberse a las diferentes competencias adquiridas por individuos con niveles similares de educación.

En este orden de ideas, Chevalier (2003), ha separado entre individuos aparentemente sobre/infra-educados, que tienen competencias similares a las de sus compañeros de trabajo correctamente educados pero con un mayor/menor nivel educativo que el requerido para el puesto de trabajo que 
ocupan. Por otro lado, se encuentran los trabajadores verdaderamente sobre-educados/infra-educados, que sufren una sobre/ infrautilización de sus competencias en el puesto de trabajo.

Otros estudios, confirman estas diferencias entre desajuste educativo y en competencias (Di Pietro \& Urwin, 2006; Green \& McIntosh, 2007), poniendo de manifiesto la necesidad de considerar la heterogeneidad en competencias entre individuos con niveles similares de educación y de considerar el grado desajuste en competencias, al analizar los efectos económicos del desajuste entre el capital humano que poseen los individuos y la demanda del mercado de trabajo.

En este orden de ideas, una revisión sistemática de la literatura empírica encontrada se puede obtener los siguientes hechos estilizados sobre las tendencias del desajuste educativo en las características de los trabajadores. (Hartog, 2000; Mora, 2005; Castillo, 2007)

a. El desajuste educativo puede ser un fenómeno temporal o permanente.

b. La tasa de retorno de la educación de los sobre-educados es mayor que la de los infra-educados, pero menor en comparación con la de los adecuadamente educados.

c. Los sobre-educados tienen una menor experiencia que los infra-educados.

d. Los sobre-educados tienden a cambiar fácilmente de ocupación.

e. Los sobre-educados tienen contratos incompletos y más cortos que los infraeducados.

f. Existe movilidad entre las personas que tienen un nivel de capital humano más bajo.

g. Existen diferencias significativas en las estimaciones, dependiendo del método que se utilice.
De esta exploración, se pueden destacar desde el punto de vista empírico, tres maneras de determinar si un individuo presenta un desajuste en el mercado laboral: el método estadístico, el método subjetivo y el método objetivo.

El método estadístico define, el desajuste de un grupo ocupacional a partir de la media más o menos una desviación estándar. Un individuo está sobre-educado si tiene más años de educación que la media más la desviación estándar del grupo de referencia y viceversa para los infra-educados.

La anterior interpretación implica que un trabajador sólo puede pasar de estar sobre o infra educado a estar correctamente educado si cambia de ocupación, aunque Dorn \& Souza-Posa (2005), muestran que es posible que un individuo mejore el emparejamiento o lo empeore desempeñando otro trabajo dentro del mismo grupo de ocupación o ajustando los requisitos del trabajo actual.

El principal inconveniente de este método es la fijación del valor de referencia relacionado al nivel educativo medio de la ocupación a tratar. Por ejemplo, si una trabajo tiene una alta proporción de trabajadores sobre-educados, se aumenta el promedio ocupacional y, por ende, el valor de referencia, de modo que se subestima el nivel de sobre-educación (McGuinness, 2006, 396). Esta falencia, ha sido expuesta principalmente por Groot \& Van den Brink (2000), que en un análisis de 25 ocupaciones, la sobre-educación "verdadera" era del 26\%, mientras que la estimada con la media más la desviación estándar dio solo el 12\%. El segundo problema es la distribución de los niveles educativos dentro de la población.

Si hay un número sistemáticamente repetido, conocido como distribución bimodal, la definición de la sobre-educación a partir de la media más la desviación estándar tendrá errores de medición. Para corregir los anteriores problemas Kiker et al. (1997) para Portugal; Ng (2001), para Hong Kong; Rubb 
\& Quinn (2002), para México, definen el desajuste educativo a partir de la mediana de la distribución salarial, que también se enmarca en el método estadístico. No obstante, a pesar de sus limitaciones el método estadístico es el más implementado por su facilidad de cálculo y disponibilidad de datos estadísticos. (Hartog, 2000)

El método subjetivo, consiste en preguntar al individuo cuáles son sus cualificaciones en el trabajo actual, con base en sus respuestas indican si se considera sobre-educado o no. Sin embargo, las medidas subjetivas tienen errores de clasificación, debido a que el investigador no sabe exactamente de qué forma el trabajador hizo inferencias acerca de su desajuste educativo.

Por último, el método objetivo, requiere conocer las habilidades y destrezas de los individuos y las habilidades y destrezas que requieren las empresas. Aunque es difícil conocer la gama de habilidades de los individuos y si hay sustitución entre ellas, este método requiere alguna información sobre las empresas.

Las investigaciones empíricas en Latinoamérica se han realizado diferentes ejercicios en Uruguay (Bucheli \& Casacuberta, 2001; Espino, 2013); Colombia (Caicedo, 2007; Castillo, 2007; Mora, 2008); México (Rubb \& Quinn, 2002) y Argentina (Pérez, 2005; Waisgrais, 2005). En este orden de ideas, en pro de contribuir a la literatura internacional de la experiencia de este fenómeno en México, este trabajo estudia las causas y efectos del desajuste educativo en el mercado laboral mexicano, entendido como la diferencia por exceso o defecto, entre el nivel educativo alcanzado por el individuo y el exigido por el puesto de trabajo en el cual se desempeña. Para lo anterior, el trabajo se organiza en cuatro secciones incluyendo la presente. A continuación se describe la metodología implementada, en la tercera se exponen los resultados encontrados y finalmente se presentan las conclusiones.

\section{METODOLOGÍA}

\section{Fuente de datos}

En esta investigación, el análisis se basa en los datos estadísticos provenientes de los microdatos de los censos de población y vivienda dispuestos por el Instituto Mexicano de Estadísticas (INEGI) y del proyecto IPUMS de la Universidad de Minessota $^{3}$. La población total de la encuesta es de 7,051,284 personas con un rango total ajustado con factores de expansión de 101 millones personas. La Tabla 1 presenta la distribución del censo por sexo, donde los hombres participan con el 65\% de los ocupados con un total de 68 millones.

Tabla 1.

Distribución de los encuestados por sexo.

\begin{tabular}{cccc}
\hline Año & Total & Hombres & Mujeres \\
\hline $\mathbf{1 9 9 0}$ & $18,002,250$ & $13,567,810$ & $4,434,440$ \\
\hline $\mathbf{1 9 9 5}$ & $24,907,861$ & $16,726,047$ & $8,181,814$ \\
\hline $\mathbf{2 0 0 0}$ & $26,089,643$ & $17,493,021$ & $8,596,622$ \\
\hline $\mathbf{2 0 1 0}$ & $32,166,127$ & $20,564,433$ & $11,601,694$ \\
\hline Total & $101,165,881$ & $68,351,311$ & $32,814,570$ \\
\hline
\end{tabular}

Fuente: Encuestas de Empleo México 1990-2010. INEGI

\section{Modelo para estimar \\ las causas del desajuste educativo}

El fenómeno de estudio, la sobre o infraeducación, es aproximada empíricamente como la diferencia entre el grado educativo del individuo y la media más o menos una desviación estándar. Se proponen modelos que hallen los determinantes de que las personas se encuentren en los estados del desajuste educativo frente a los que están correctamente educados.

\footnotetext{
${ }^{3}$ Para más información del proyecto ir a la página https://international.ipums.org/international/
} 
Estadísticamente este tipo de variables se categoriza en cada uno en el caso de que la persona este sobre-educada/ infraeducada y cero en caso contrario. Cuando las variables de análisis poseen estas características, de poseer únicamente dos valores, se denominan variables dicotómicas y en su estudio se implementan los modelos de elección discreta.

En general, se considera que detrás de la variable dependiente en estos modelos, $Y$, se encuentra una variable no observable, $I$, que depende de un conjunto de variables explicativas $X_{i}$ que toma ciertos valores si ha sobre pasado cierto lumbral, como se expresa a continuación.

$$
Y_{i}=\left\{\begin{array}{l}
1 \text { si } I_{i}^{*}>0 \text { lo que ocurre cuando } X_{i} \beta+\mathcal{E}_{i}>0 \\
0 \text { si } I_{i}^{*}<0 \text { lo que ocurre cuando } X_{i} \beta+\mathcal{E}_{i}<0
\end{array}\right.
$$

Donde el supuesto sobre la distribución de, determina el tipo de modelo a estimar: si se supone una función de distribución uniforme, se utiliza el modelo lineal de probabilidad truncado; si se distribuye como una normal con media cero y varianza uno, el modelo generado será un probit; mientras que si se supone que es mediante una curva logística, se trataría de un modelo logit. La hipótesis de que el umbral a superar por la variable latente sea cero, se puede modificar por cualquier otro valor sugiriéndose que el valor crítico sea el definido por el término constante (Pérez, 2005). Bajo el primer enfoque el modelo probabilístico quedaría definido (Medina, 2003)

$$
\begin{aligned}
& P_{i}=\operatorname{Pr} o b\left(Y_{i}=1\right)=\operatorname{Pr} o b\left(I_{i}^{>}>0\right)= \\
& \operatorname{Pr} o b\left(X_{i} \beta+\mathcal{E}_{i}>0\right)=F\left(X_{i} \beta\right)
\end{aligned}
$$

La efectividad de estos modelos se miden a través de dos indicadores: el primero es el grado de la varianza de la variable dependiente captada o predicha por parte de las variables independientes, indicador denominado $\mathrm{R}^{2}$. Entre más alto, más efectivo es el modelo en determinar el comportamiento de la variable dependiente y viceversa. El segundo es el grado de observaciones del modelo que han sido clasificadas correctamente en la característica analizada, un mayor porcentaje indica que el grado de robustez del modelo es mejor. En términos estadísticos, hay diferentes situaciones sobre la naturaleza de los datos que se deben corregir para una óptima calibración del modelo tales como la presencia de heterocedasticidad, multicolinealidad y autocorrelacion serial (Pérez, 2005). Tomando en cuenta los criterios anteriores, se propone para este trabajo la utilización de la siguiente función probabilística:

$$
\begin{aligned}
& Y=f(S, X) \\
& Y=a+\beta_{i} X_{i}+\mathcal{E}
\end{aligned}
$$

Donde es la variable a explicar o dependiente categorizada de forma dicotómica, donde 1 es cuando la persona este sobreeducada/infra-educada y cero en caso contrario. Por su parte, $\beta$, captan el aporte marginal de cada uno de estos factores a la probabilidad de estar sobre-educado/ infra-educado, mientras que $\mathcal{E}$ es el error de la estimación que se distribuye según la función probabilística. Esta última es explicada por un vector que resume factores característicos de la persona, $X$, que se seleccionaron con base a las hipótesis planteadas:

H1: Ser mujer aumenta la probabilidad de estar infra-educado. (Hartog, 2000; Mora, 2005; Castillo, 2007)

H2: Los individuos que poseen mayores grados educativos tienden a estar más sobre-educados a la vez que suelen tener menos experiencia. (Hartog, 2000) 


\section{Modelo para estimar el efecto del desajuste en los ingresos laborales}

Para evaluar el efecto del desajuste educativo en el mercado laboral, se estimará el ingreso laboral por hora de los diferentes tipos de trabajadores, mediante el modelo de Mincer (1974). Esta especificación involucra las características de los trabajadores como determinantes de los ingresos laborales de los ocupados, es uno de los modelos más explorados y conocidos en la literatura sobre economía laboral. (Psacharopoulos, 2004; Cohn \& Addison, 1998; Asplund \& Pereira, 1999; Harmon, Walker \& Nielsen, 2001). Este modelo parte de una función semilogarítmica (ecuación 1) que se estima por medio del procedimiento de Mínimos Cuadrados Ordinarios (MCO), teniendo como variable dependiente el logaritmo de los salarios de los trabajadores y como variables independientes los años de educación del trabajador, la experiencia laboral (y su cuadrado), la rama de actividad, su ocupación y la tenencia de contrato.

$$
\begin{aligned}
\log (\mathrm{Y})= & \beta_{0}+B_{1} \operatorname{Educ}+B_{2} \operatorname{Exp}+ \\
& B_{3} \operatorname{Exp} 2+B_{4} \mathrm{~S}+B_{5} \mathrm{~L}+\mathcal{\varepsilon}
\end{aligned}
$$

- $Y$ es el ingreso laboral por hora del individuo

- Educ es el nivel educativo.

- Exp son los años de experiencia laboral

- $L$ corresponde al desajuste educativo presentado por el trabajador.

- $\varepsilon$ es el término de perturbación aleatoria que se distribuye según una normal

Entre las otras variables del trabajador que se van a controlar en este estudio, están la provincia donde habita el individuo, estado civil, etnia, entre otras. En términos de interpretación, los coeficientes (B) representan la variación porcentual sobre el ingreso laboral tendrá sobre cada uno de los cambios sobre las características de los tra- bajadores. La efectividad de este modelo se mide a través de la varianza de la variable dependiente captada por parte de las variables de control. Este indicador se denomina $\mathrm{R}^{2}$. Si este es más alto, más efectivo es el modelo en determinar el comportamiento de la variable dependiente. Hay diferentes situaciones sobre la naturaleza de los datos que también se deben arreglar para una correcta calibración del modelo tales como la presencia de heterocedasticidad, multicolinealidad y autocorrelacion serial.

Asimismo, hay que corregir un problema adicional que se encuentra implícito en los datos, el denominado sesgo de selección. Lo que consiste en la ausencia de aleatoriedad muestral, o selección muestral no aleatoria dentro de las encuestas de hogares que se realizan (De Oliveira \& Ariza, 2000). Se denomina sesgo, porque una muestra que está concentrada en cierto grupo poblacional desvía el resultado obtenido de una variable de interés hacia el grupo que tenga mayor representación. Este problema es necesario de corregir cuando se investiga sobre diferencias en los ingresos laborales de las personas sobre grupos de población específicos, ya que no permite obtener resultados exactos del fenómeno analizado.

La corrección de Heckman es la solución más utilizada en la literatura para efectos de estimación (Heckman, 1979; De Oliveira \& Ariza, 2000). Se desarrolla, estimando dos regresiones, la primera se realiza a base de la ecuación de participación, entendida como la probabilidad de que el individuo participe en el mercado laboral contra los factores que pueden incidir en la elección.

$$
\mathrm{pi}=\text { Bo }+\mathrm{zi} \varphi+\mathrm{ui}
$$

Donde pi se refiere a la probabilidad de participar en el mercado laboral, zi corresponde a un vector con variables explicativas que influyen en la decisión de participar, $\varphi$ es un vector de parámetros, y ui son los 
errores. Después de realizada, el segundo cálculo es la ecuación de Mincer incorporando la corrección de selección realizada por lambda $(\lambda)$ como inversa del ratio de Mill. (De Oliveira \& Ariza, 2000)

$$
\log (\mathrm{Y})=\quad \begin{aligned}
& \quad B 0+B 1 \mathrm{~S}+62 \operatorname{Exp}+ \\
& \quad B 3 \operatorname{Exp} 2+\beta 4 \mathrm{~L}+\lambda \operatorname{ti} \theta+\varepsilon \mathrm{i}
\end{aligned}
$$

Con ello se ha corregido el sesgo de selección. Usando el argumento de Torres \& Celton (2009), la importancia del uso del $(\lambda)$ se determina al estimar la regresión, siempre y cuando su coeficiente sea significativo. En el caso de no ser representativo, se podría trabajar directamente sin la necesidad de la corrección. Para este tipo de cálculo se quieren indagar sobre las hipótesis ofrecidas en la literatura:
H3: Los sobre-educados ganan más que los infra-educados, pero ganan menos que los que están adecuadamente educados (Verdugo y Verdugo; 1989). Sin embargo, también se ha encontrado que hay retornos positivos a la sobre-educación y negativos a la infra-educación. (Cohn \& Kahn, 1995; Duncan \& Hoffman, 1981)

H4: Se encuentran diferencias significativas entre los retornos para sobre-educados en las diferentes ocupaciones. Verdugo \& Verdugo (1988); Cohn \& Kahn (1995) y Kiker, Santos \& Oliveira (1997)

\section{RESULTADOS}

La Tabla 2 expone las estadísticas descriptivas relacionadas con la distribución de trabajadores en la base total de empleo.

Tabla 2.

Porcentaje de trabajadores por tipo de desajuste laboral. Estadísticas descriptivas. Año 2010

\begin{tabular}{|c|c|c|c|c|c|}
\hline \multicolumn{2}{|r|}{ Variable } & Desajuste & $\begin{array}{l}\text { Infra- } \\
\text { educado }\end{array}$ & $\begin{array}{c}\text { Sobre- } \\
\text { educado }\end{array}$ & Correctamente \\
\hline \multicolumn{2}{|r|}{ Total } & 31.0 & 14.8 & 16.2 & 69.0 \\
\hline \multirow{2}{*}{ Sexo } & Hombre & 31.8 & 14.7 & 68.2 & 17.1 \\
\hline & Mujer & 29.6 & 15.1 & 70.4 & 14.5 \\
\hline \multirow{3}{*}{ Sector } & Primario & 40.4 & 20.0 & 59.6 & 20.4 \\
\hline & Secundario & 30.1 & 14.8 & 69.9 & 15.3 \\
\hline & Terciaria & 29.1 & 13.6 & 70.9 & 15.5 \\
\hline \multirow{5}{*}{ Edad } & Menos de 25 & 26.9 & 6.9 & 73.1 & 20.0 \\
\hline & 25 a 35 & 27.7 & 8.9 & 72.3 & 18.8 \\
\hline & 35 a 45 & 28.3 & 12.4 & 71.7 & 15.9 \\
\hline & 45 a 55 & 34.5 & 21.2 & 65.5 & 13.3 \\
\hline & 55 o más & 47.1 & 39.6 & 52.9 & 7.5 \\
\hline \multirow{3}{*}{ Educación } & Primaria & 13.1 & 6.8 & 86.9 & 6.3 \\
\hline & Secundaria & 43.0 & 3.5 & 57.0 & 39.5 \\
\hline & Universitario & 40.7 & 0.4 & 59.3 & 40.3 \\
\hline \multirow{4}{*}{ Área } & Hasta 2,499 habitantes & 34.8 & 19.8 & 65.2 & 15.0 \\
\hline & 2,500 a 14,999 & 31.9 & 16.7 & 68.1 & 15.2 \\
\hline & 15,000 a 99,999 & 30.6 & 14.5 & 69.4 & 16.1 \\
\hline & 100,000 o mas & 29.4 & 12.4 & 70.6 & 16.9 \\
\hline \multicolumn{2}{|c|}{ Observaciones expandidas } & $11,929,770$ & $5,703,465$ & $6,226,305$ & $26,556,653$ \\
\hline
\end{tabular}

Fuente: cálculos del autor con base en los censos de población en México 2010. INEGI 
Se encontró que el grado de desajuste laboral afecta al 31\% de los mexicanos ocupados, con el 16\% sobre-educados y $15 \%$ infra-educados. Por género el desajuste afecta ligeramente más a los hombres con el $32 \%$ frente a las mujeres con el 29,6\%. Por sector económico, el 40\% los trabajadores del primario presenta desajuste educativo contra $30 \%$ secundario y terciario (29\%). Igualmente los trabajadores con una mayor dotación de capital humano (aproximado por edad o grado educativo), suelen padecer el desajuste, especialmente sobredotación, por ejemplo un $40 \%$ de los trabajadores con grado universitario parecen estar sobre-educados para su puestos contra un $6.3 \%$ de los de primaria, evidencia que es común para los países en desarrollo, donde la falta de oportunidades laborales en sectores que demanden a trabajadores con alta dotación de capital humano es común. (Rubb \& Quinn, 2002)

El seguimiento inter-temporal del desajuste educativo expuesto en la figura 1 del mercado laboral mexicano, indica que no han sucedido cambios significativos entre el 1990 y 2010 manteniéndose alrededor del 31\%. Sin embargo, hay una leve reducción de los niveles de infra-educado $(-2 \%)$ y un aumento de los sobre educados $(+2 \%)$.

Sobre el modelo para estimar las causas del desajuste educativo se seleccionaron 594,972 individuos para la regresión con infra-educados y 613,000 individuos para sobre-educados. Posteriormente se realizaron las pruebas de chi cuadrado para probar el tipo de función con el cual se distribuía la variable dependiente, encontrándose que para ambos ejercicios era mediante una representación de una curva logística, sin embargo, también se decidió calcular el modelo suponiendo una distribución de tipo probit.

En este orden de ideas, la Tabla 3, muestra los resultados de las estimaciones de los modelos logit con los coeficientes y elasticidades (efecto marginal) de cada una de las variables de control (independientes) sobre la probabilidad del individuo a estar sobre-educado o infra-educado entre 1990 y el 2010.

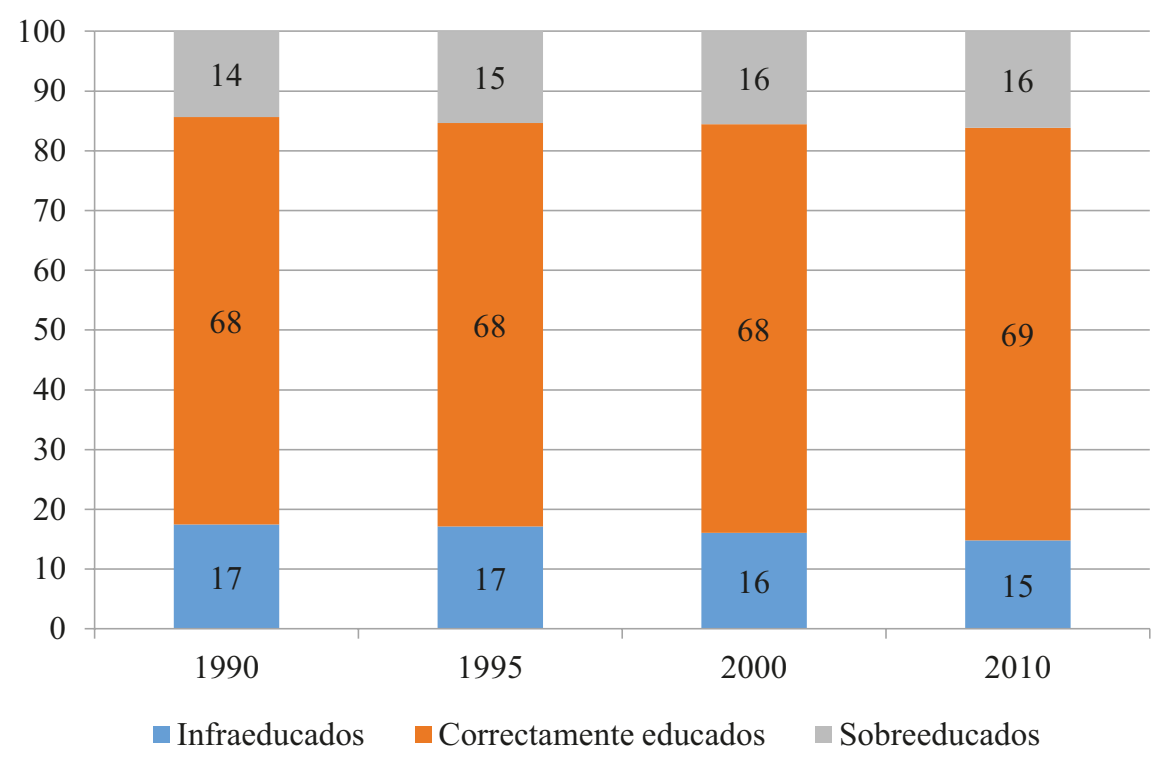

Figura 1. Porcentaje de desajuste educativo por tipo y año. Años 1990-1995-2000-2010 
Tabla 3.

Estimaciones modelo logit sobre probabilidad de ser infra educado o sobre educado

\begin{tabular}{|c|c|c|c|c|c|c|c|c|}
\hline \multirow[b]{2}{*}{ Variable } & \multirow{2}{*}{$\begin{array}{c}\text { Característica } \\
\text { Evaluada }\end{array}$} & \multicolumn{3}{|c|}{ Sobre-educados } & \multicolumn{3}{|c|}{ Infra-educados } & \multirow{2}{*}{$\begin{array}{c}\text { Característica } \\
\text { Base }\end{array}$} \\
\hline & & $\begin{array}{c}\text { Efecto } \\
\text { marginal }\end{array}$ & Error & $\mathrm{p}(\mathrm{z})$ & $\begin{array}{c}\text { Efecto } \\
\text { marginal }\end{array}$ & Error & $\mathrm{p}(\mathrm{z})$ & \\
\hline \multicolumn{2}{|c|}{ Edad (proxy experiencia) } & $-0.03 \%$ & 0.0000 & 0 & $0.1 \%$ & 0.0000 & 0 & Continua \\
\hline $\begin{array}{l}\text { Jefe de } \\
\text { hogar }\end{array}$ & $\mathrm{Si}$ & $-1.4 \%$ & 0.0010 & 0 & $2.3 \%$ & 0.0009 & 0 & No \\
\hline Sexo & Mujer & $-1.3 \%$ & 0.0009 & 0 & $2.5 \%$ & 0.0010 & 0 & Hombre \\
\hline \multirow{3}{*}{$\begin{array}{c}\text { Nivel } \\
\text { educativo }\end{array}$} & Primaria & $5.0 \%$ & 0.0168 & 0 & $-0.6 \%$ & 0.0014 & 0 & \multirow{3}{*}{ Ninguno } \\
\hline & Secundaria & $9.5 \%$ & 0.0009 & 0 & $-4.3 \%$ & 0.0008 & 0 & \\
\hline & Universitaria & $19.0 \%$ & 0.0012 & 0 & $-3.8 \%$ & 0.0007 & 0 & \\
\hline \multirow{8}{*}{ Ocupación } & Profesionales & $-1.6 \%$ & 0.0016 & 0 & $1.8 \%$ & 0.0039 & 0 & \multirow{8}{*}{ Directores } \\
\hline & Técnicos & $10.1 \%$ & 0.0065 & 0 & $-5.3 \%$ & 0.0017 & 0 & \\
\hline & $\begin{array}{l}\text { Empleados de } \\
\text { oficina }\end{array}$ & $19.1 \%$ & 0.0092 & 0 & $-7.0 \%$ & 0.0012 & 0 & \\
\hline & $\begin{array}{l}\text { Trabajadores } \\
\text { de servicios y } \\
\text { vendedores }\end{array}$ & $48.6 \%$ & 0.0132 & 0 & $-14.5 \%$ & 0.0011 & 0 & \\
\hline & $\begin{array}{l}\text { Trabajadores } \\
\text { agrícolas } \\
\text { calificados }\end{array}$ & $71.8 \%$ & 0.0122 & 0 & $-22.6 \%$ & 0.0021 & 0 & \\
\hline & $\begin{array}{c}\text { Operarios de la } \\
\text { industria }\end{array}$ & $61.5 \%$ & 0.0128 & 0 & $-16.1 \%$ & 0.0011 & 0 & \\
\hline & $\begin{array}{l}\text { Operadores de } \\
\text { máquinas y } \\
\text { ensambladores }\end{array}$ & $65.4 \%$ & 0.0118 & 0 & $-11.1 \%$ & 0.0008 & 0 & \\
\hline & $\begin{array}{c}\text { Trabajadores no } \\
\text { calificados }\end{array}$ & $77.1 \%$ & 0.0097 & 0 & $-15.4 \%$ & 0.0010 & 0 & \\
\hline \multirow{2}{*}{ Rama } & Industria & $-3.0 \%$ & 0.0019 & 0 & $4.5 \%$ & 0.0027 & 0 & \multirow{2}{*}{ Agricultura } \\
\hline & Servicios & $-5.2 \%$ & 0.0030 & 0 & $5.7 \%$ & 0.0023 & 0 & \\
\hline \multirow{6}{*}{ Año } & 1995 & $0.4 \%$ & 0.0024 & 0.1 & $1.1 \%$ & 0.0035 & 0.002 & \multirow{3}{*}{1990} \\
\hline & 2000 & $-2.1 \%$ & 0.0013 & 0 & $3.7 \%$ & 0.0011 & 0 & \\
\hline & 2010 & $-4.7 \%$ & 0.0026 & 0 & $8.4 \%$ & 0.0012 & 0 & \\
\hline & Observaciones & \multicolumn{3}{|c|}{594,972} & \multicolumn{2}{|c|}{613,000} & & \\
\hline & $\mathrm{R} 2$ & \multicolumn{3}{|c|}{0.385} & \multicolumn{2}{|c|}{0.393} & & \\
\hline & $\begin{array}{l}\text { Clasificación } \\
\text { correcta }\end{array}$ & \multicolumn{3}{|c|}{$87.6 \%$} & \multicolumn{2}{|c|}{$84.0 \%$} & & \\
\hline
\end{tabular}

Fuente: cálculos del autor con base en los censos de población México 1990-2010. INEGI

En términos del ajuste global, el modelo resulta aceptable considerando que el valor del estadístico $\mathrm{chi}^{2}$ es muy significativo siendo la probabilidad de que la combinación lineal del modelo no sea significativo igual a cero. A su vez, todas las variables introducidas presentaron niveles de significancia estadística del 5\% y según el R2 ajustado, los modelos consiguen explicar entre un $38.5 \%$ y $39.3 \%$ de la varianza de las variables dependientes, mientras que el porcentaje de casos correctamente clasi- 
ficados dentro del modelo propuesto alcanzó cerca del 85\%. Con esta verificación de robustez del modelo, se pasa a comprobar cada una de las hipótesis propuestas por el trabajo.

Para la interpretación de los resultados, el efecto marginal (elasticidad) en las variables continuas, equivale al porcentaje en el que se incrementa la probabilidad de estar sobre-educado/infra-educado dado un cambio del $1 \%$ en la variable independiente de análisis. Para las categóricas, el efecto marginal mide la probabilidad de manera relativa como comparación de una característica base. En la hipótesis inicial de la literatura, se evidencia que el ser mujer aumenta la probabilidad de estar infra-educado (Hartog, 2000; Mora, 2005; Castillo, 2007), las mexicanas tienen un $2.5 \%$ de probabilidad adicional de estar infra-educadas en su ocupación en relación a los hombres.

En términos de nivel educativo, se observa que los individuos que poseen mayores dotación de capital humano tienden a estar más sobre-educados (Hartog, 2000), pero menor de estar infra-educados. Por ejemplo, los trabajadores con postgrado tienen un $19 \%$ de probabilidad de estar sobre califica- dos que lo que no tienen ningún nivel educativo, asimismo tienen un $3.8 \%$ de probabilidad menor de estar infra-educados. En términos de experiencia, los trabajadores sobre-educados, a pesar de ser más educados, suelen ser menos experimentados, de hecho un año adicional de edad disminuye la probabilidad de ser sobre-cualificado en un $-0.2 \%$.

Lo anterior puede confirmar, que los mexicanos con altos nieles educativos escogen trabajados que les permiten acumular experiencia para luego obtener mejores puestos de trabajo. Otros resultados interesantes, obtenidos en el ejercicio son la disminución año a año en la probabilidad de ser sobre-educado contra el aumento de ser infra-educado.

En este sentido, los trabajadores de 2010 tuvieron un $-4.7 \%$ de ser sobre-educados que los ubicados en 1990 y un 8.4\% más en ser infra-educados. A continuación se procedió a realizar la ecuación de Mincer con corrección de heckman para el efecto del desajuste educativo en los ingresos laborales por hora para cada uno de los años censales disponibles (1990-1995-2000-2010), cuyos resultados se muestran en la Tabla 4.

Tabla 4.

Resultado estimaciones sobre el ingreso laboral por hora mediante corrección de Heckman.

Años 1990 - 1995 - $2000-2010$

\begin{tabular}{|c|c|c|c|c|c|c|}
\hline Variable & Característica evaluada & 1990 & 1995 & 2000 & 2010 & $\begin{array}{c}\text { Característica } \\
\text { base }\end{array}$ \\
\hline \multirow{2}{*}{ Desajuste } & Correctamente educados & $3.0 \%$ & $11.4 \%$ & $4.0 \%$ & $1.4 \%$ & \multirow{2}{*}{ Infra-educado } \\
\hline & Sobre-educado & $5.9 \%$ & $24.4 \%$ & $4.6 \%$ & $1.5 \%$ & \\
\hline $\begin{array}{l}\text { Jefe de } \\
\text { hogar }\end{array}$ & $\mathrm{Si}$ & $8.7 \%$ & $7.6 \%$ & $8.5 \%$ & $6.7 \%$ & No \\
\hline Sexo & Mujer & $-8.6 \%$ & $-4.4 \%$ & $-7.3 \%$ & $-7.5 \%$ & Hombre \\
\hline \multirow{4}{*}{ Edad } & 25 a 35 & $14.2 \%$ & $20.0 \%$ & $15.7 \%$ & $12.6 \%$ & \multirow{4}{*}{ Menores 25} \\
\hline & 36 a 45 & $20.3 \%$ & $22.8 \%$ & $21.1 \%$ & $17.8 \%$ & \\
\hline & 46 a 55 & $20.3 \%$ & $23.1 \%$ & $20.6 \%$ & $18.7 \%$ & \\
\hline & 56 en adelante & $9.7 \%$ & $10.1 \%$ & $10.4 \%$ & $10.1 \%$ & \\
\hline
\end{tabular}


Cont...

\begin{tabular}{|c|c|c|c|c|c|c|}
\hline Variable & Característica evaluada & 1990 & 1995 & 2000 & 2010 & $\begin{array}{c}\text { Característica } \\
\text { base }\end{array}$ \\
\hline \multirow{3}{*}{ Educación } & Primaria & $15.8 \%$ & $14.6 \%$ & $15.0 \%$ & $16.6 \%$ & \multirow{3}{*}{ Ninguno } \\
\hline & Secundaria & $35.7 \%$ & $26.3 \%$ & $44.0 \%$ & $40.2 \%$ & \\
\hline & Universitaria & $59.7 \%$ & $37.4 \%$ & $75.3 \%$ & $70.2 \%$ & \\
\hline Inmigrante & $\mathrm{Si}$ & $28.5 \%$ & $40.2 \%$ & $31.8 \%$ & $24.9 \%$ & No \\
\hline \multirow{11}{*}{$\begin{array}{l}\text { Rama de } \\
\text { actividad }\end{array}$} & Minería & $43.3 \%$ & $44.4 \%$ & $27.4 \%$ & $39.6 \%$ & \multirow{11}{*}{ Agricultura } \\
\hline & Industria & $18.6 \%$ & $13.0 \%$ & $9.6 \%$ & $1.2 \%$ & \\
\hline & Electricidad, gas y agua & $34.7 \%$ & $36.3 \%$ & $37.4 \%$ & $33.1 \%$ & \\
\hline & Construcción & $21.3 \%$ & $13.2 \%$ & $14.5 \%$ & $17.4 \%$ & \\
\hline & Comercio & $19.3 \%$ & $16.2 \%$ & $5.1 \%$ & $1.9 \%$ & \\
\hline & Hoteles y restaurantes & $14.1 \%$ & $13.4 \%$ & $4.4 \%$ & $7.5 \%$ & \\
\hline & Transporte y comunicaciones & $29.5 \%$ & $18.6 \%$ & $12.1 \%$ & $4.0 \%$ & \\
\hline & Servicios financieros y seguros & $40.5 \%$ & $43.5 \%$ & $36.5 \%$ & $27.3 \%$ & \\
\hline & $\begin{array}{c}\text { Administración pública y } \\
\text { defensa }\end{array}$ & $11.0 \%$ & $32.7 \%$ & $14.9 \%$ & $19.2 \%$ & \\
\hline & Educación & $30.8 \%$ & $41.3 \%$ & $36.7 \%$ & $42.5 \%$ & \\
\hline & otros servicios & $18.7 \%$ & $16.0 \%$ & $6.2 \%$ & $3.5 \%$ & \\
\hline \multirow{8}{*}{ Ocupación } & Profesionales & $-17.2 \%$ & $25.4 \%$ & $-18.9 \%$ & $-6.3 \%$ & \multirow{8}{*}{ Directores } \\
\hline & Técnicos no universitarios & $-41.7 \%$ & $-14.7 \%$ & $-37.9 \%$ & $-16.6 \%$ & \\
\hline & Empleados de oficina & $-46.6 \%$ & $-28.1 \%$ & $-47.9 \%$ & $-38.7 \%$ & \\
\hline & Vendedores & $-65.7 \%$ & $-58.4 \%$ & $-69.6 \%$ & $-51.3 \%$ & \\
\hline & Obreros agropecuarios & $-86.3 \%$ & $-81.1 \%$ & $-79.1 \%$ & $-58.1 \%$ & \\
\hline & Operarios de la industria & $-57.9 \%$ & $-45.1 \%$ & $-63.6 \%$ & $-37.4 \%$ & \\
\hline & Operadores de maquinas & $-62.0 \%$ & $-44.0 \%$ & $-64.1 \%$ & $-43.7 \%$ & \\
\hline & Trabajadores no calificados & $-73.1 \%$ & $-53.1 \%$ & $-72.2 \%$ & $-51.8 \%$ & \\
\hline \multirow{3}{*}{$\begin{array}{l}\text { Tamaño } \\
\text { ciudad }\end{array}$} & 2.500 a 14.999 habitantes & $14.7 \%$ & $6.3 \%$ & $5.3 \%$ & $5.1 \%$ & $\begin{array}{c}1 \text { a } 2,499 \\
\text { habitantes }\end{array}$ \\
\hline & 15000 a 99999 & $17.8 \%$ & $10.3 \%$ & $7.9 \%$ & $8.2 \%$ & \\
\hline & 100.000 o mas & $21.5 \%$ & $22.5 \%$ & $16.9 \%$ & $15.0 \%$ & \\
\hline \multirow{3}{*}{$\begin{array}{c}\text { Clase de } \\
\text { trabajador }\end{array}$} & Cuenta propia & $-31.7 \%$ & $-41.0 \%$ & $-34.0 \%$ & $-26.4 \%$ & \multirow{3}{*}{ Empleador } \\
\hline & Empleado o obrero & $-46.2 \%$ & $-54.0 \%$ & $-50.2 \%$ & $-36.5 \%$ & \\
\hline & Jornalero & $-29.7 \%$ & $-34.6 \%$ & $-50.9 \%$ & $-32.4 \%$ & \\
\hline & Constante & 8.2324 & 1.8778 & 3.0505 & 3.4221 & \\
\hline & Observaciones & $17,642,390$ & $24,389,234$ & $25,645,373$ & $31,549,220$ & \\
\hline & R2 & 0.2343 & 0.4053 & 0.4273 & 0.3302 & \\
\hline
\end{tabular}

Fuente: cálculos del autor con base en los censos de población Mexico 1990-2010. INEGI 
El número total de trabajadores fue de 99.2 millones con un grado de varianza de promedio del $35 \%$. El modelo es logarítmico así que el cambio de un $1 \%$ en las variables continuas se interpreta como el cambio porcentual en la variable dependiente.

Igualmente las variables categóricas, indican un cambio porcentual con relación a una característica base. Se aprecia que existe evidencia estadística que respalda que hay retornos positivos al sobre-educación y negativos a la infraeducación (Cohn \& Kahn, 1995; Duncan \& Hoffman, 1981). En promedio los infraeducados ganan un $4.95 \%$ menos que los correctamente educados mientras que los sobre-educados ganan un $9.1 \%$ más. Se aprecia un efecto significativamente volátil en el año de 1995 cuando la ganancia de los correctamente educados aumenta al $11 \%$ y de los sobre-educados al $24 \%$.

Estos resultados pueden deberse a la crisis económica sufrida por México a causa de la crisis del tequila en este año, forzando a una caída de los salarios. Este movimiento, afecto más negativamente a los trabajadores de bajos ingresos ampliando la brecha salarial entre grupos.

Lo anterior, muestra que el desajuste educativo es un fenómeno que puede ser afectado por el ciclo económico, aspecto que no ha sido estudiado en detalle por la literatura. En la Tabla 4 se muestran los ejercicios sobre las estimaciones de los retornos por tipo de ocupación. Se encuentran diferencias significativas entre los retornos para sobre-educados en las diferentes ocupaciones tal y como se encuentra en Verdugo \& Verdugo (1988); Cohn \& Kahn (1995) y Kiker, Santos \& Oliveira (1997).

Asimismo, se aprecia que son en las ocupaciones donde se requiere mayor capital humano donde se observan las mayores diferencias. Por ejemplo, los profesionales correctamente educados ganan cerca de un $22 \%$ que los infra-educados y los sobreeducados un 27,7\%. En los técnicos no universitarios el diferencial es de 11,6\% y de $20,3 \%$ respectivamente. Por su parte, los empleados de oficina, operadores de maquinaria e industriales no cuentan con un diferencial elevado (ver Tabla 5).

Tabla 5.

Ganancias sobre el ingreso laboral por hora de los infra-educados por ocupación. Años 1990-2010

\begin{tabular}{ccc}
\hline Ocupación & Correctamente & $\begin{array}{c}\text { Sobre } \\
\text { educado }\end{array}$ \\
\hline Profesionales & $22.64 \%$ & $27.73 \%$ \\
\hline $\begin{array}{c}\text { Técnicos no } \\
\text { universitarios }\end{array}$ & $11.68 \%$ & $20.35 \%$ \\
\hline $\begin{array}{c}\text { Empleados de } \\
\text { oficina }\end{array}$ & $0.29 \%$ & $4.89 \%$ \\
\hline $\begin{array}{c}\text { Vendedores } \\
\text { Obreros }\end{array}$ & $6.77 \%$ & $10.08 \%$ \\
\hline $\begin{array}{c}\text { agropecuarios } \\
\text { Operarios de la } \\
\text { industria }\end{array}$ & $10.69 \%$ & $16.57 \%$ \\
\hline $\begin{array}{c}\text { Operadores de } \\
\text { maquinas }\end{array}$ & $0.97 \%$ & $2.41 \%$ \\
\hline $\begin{array}{c}\text { Trabajadores no } \\
\text { calificados }\end{array}$ & $0.80 \%$ & $2.10 \%$ \\
\hline
\end{tabular}

Fuente: cálculos del autor con base en los censos de población Mexico 1990-2010. INEGI

Entre otros resultados de interés se aprecia que las mujeres ganan en promedio un $7 \%$ menos que los hombres por causa de la discriminación salarial de género que existe en el México (De Oliveira \& Ariza, 2000). En términos de edad existe una ganancia creciente a medida que se crece ya que se relaciona con lo anterior con un mayor capital humano informal, de hecho en promedio las personas de 36 a 55 años tienen salarios un $20 \%$ mayor que sus pares menores de 25 años. Sin embargo, a partir de esta edad disminuye el retorno hasta un $10 \%$ lo que muestra que la función de relación entre la edad y el salario es cóncava y negativa 
en el tiempo (Mincer, 1974). Por su parte, los jefes de hogar ganan un $7 \%$ más que los que no son. Los ocupados del sector terciario también obtienen un $30 \%$ que los del sector primario mientras que los del secundario un $25 \%$.

\section{CONCLUSIONES}

La presencia de desajustes educativos en el mercado laboral, es señal de preocupación para los agentes, ya que este desestimula la inversión en educación dado que las tasas de rendimiento esperado disminuyen (Mora, 2008). Por esto, la importancia en los países en desarrollo lograr una paridad entre los puestos que se ofrecen y la demanda de trabajadores. En este sentido, el presente trabajo evidenció la presencia de desajustes educativos estructurales en el mercado laboral mexicano cercano al $31 \%$ donde la sobreeducación es mayoritaria con el $16.2 \%$ y la infra-educación con el $14.8 \%$ que no han presentado variaciones en los 20 años de estudio.

Los resultados evidencian que existen diferencias de género en la sobre-educación. En términos de la movilidad laboral, los sobre-educados tienen menor experiencia que los infra-educados y la probabilidad de que obtengan un contrato temporal es mayor. La teoría de la competencia por puestos de trabajo se aplica en el caso de los trabajadores con menor capital humano, quienes se emplean con el fin de obtener un contrato, sin importar que haya desajuste educativo. Al estimar los rendimientos asociados al ingreso laboral por hora y el tipo de desajuste educativo, se observa, que este último contribuye a explicar parte de las diferencias salariales observadas entre trabajadores que ocupan un puesto similar, con un rendimiento positivo para los años de sobre- educación y una penalización sobre los salarios en el caso de la infra-educación.

Sin embargo, se observan diferencias entre las diferentes ocupaciones, siendo aquellas que necesitan más cualificación donde se presentan las mayores penalizaciones por tipo de desajuste. Los resultados también evidencian que los sobreeducados escogen temporalmente trabajos donde están sobre-educados, ya que buscan adquirir habilidades para desempeñarse en otras áreas. Pero la existencia de sobre-educación en todos los niveles educativos abre la discusión respecto de las competencias que se adquieren en las instituciones educativas y las competencias que son necesarias en el mercado laboral.

Esta asimetría debería llevar los países a adoptar como política la mejora de las competencias de la fuerza de trabajo modificando los sistemas educativos. El primer paso consiste en conocer dichas diferencias y luego revisar los programas de las instituciones educativas para hacerlos cada vez más pertinentes. Es en este sentido que las iniciativas de los observatorios laborales para egresados, para conocer las competencias de los egresados y las que necesitan los empresarios son un primer paso para solucionar el problema de la sobre-educación en el país.

Entre las limitaciones del estudio se encuentró la imposibilidad de estudiar el desajuste desde un punto de vista estructural ya que en la base de datos no se poseen las necesidades implícitas de las empresas. Los resultados obtenidos de estos ejercicios podrían confirmar que no solo los años de educación recibida sino también las competencias realmente adquiridas por los trabajadores inciden en la determinación de los salarios. Finalmente se encontró que el método estadístico, a pesar de sus limitaciones, arroja resultados acordes con la teoría. 


\section{REFERENCIAS}

Asplund, R. \& Telhado, P. (1999). Returns to Human Capital in Europe. Recuperado de: http://www.etla.fi/ wp-content/uploads/b156.pdf

Bucheli, F. \& Casacuberta, C. (2001). Sobre educación y prima salarial de los trabajadores con estudios universitarios en Uruguay. Recuperado de: http://www.fcs.edu.uy/archivos/ Doc0601.pdf

Castillo, M. (2007). Desajuste educativo por regiones en Colombia: ¿competencia por salarios o por puestos de trabajo? Cuadernos de Economía, 26(46), 108-145.

Chevalier, A. (2003). Measuring Overeducation. Economica, 70(279), 509531. doi: 10.1111/1468-0335.t01-100296

Chon, E. \& Addison, J. (1998). The Economics Returns to Lifelong Learning in OECD Countries. Education Economics, 6(3), 253-307. doi: 10.1080/09645299800000021

Cohn, E. \& S. Khan. (1995). The Wage Effects of Overschooling Revisited. Labour Economics, 2(1), 67-76. doi: 10.1016/0927-5371(95)80008-L

De Oliveira, O. \& Ariza, M. (2000). Género, trabajo y exclusión social en México. Estudios demográficos y urbanos, 15(43), 11-33.

Di Pietro, G. \& Urwin, P. (2006). Education and Skills Mismatch in the Italian Graduate Labor Market. Applied Economics, 38(1), 79-93. doi: 10.1080/00036840500215303

Dorn, D. \& A. Souza-Poza. (2005) Overqualification: Permanent or Transitory, paper presented at the International Conference on Educational
Economics. Recuperado de: http:// citeseerx.ist.psu.edu/viewdoc/down load?doi=10.1.1.172.8623\&rep=rep1 \&type $=\mathrm{p}$

Duncan, G. \& Hoffman, S. D. (1981), The Incidence and Wage Effects of Overeducation. Economics of Education Review, 1(1), 75-86. doi: 10.1016/0272-7757(81)90028-5

Durán, J. \& Mora, J. J. (2006). Una aproximación empírica a la relación entre el desempleo y las vacantes para Popayán, 2001-2005, Lecturas de Economía, 65(2), 209-222.

Espino, A. (2013). Brechas salariales en Uruguay: género, segregación y desajustes por calificación. Problemas del desarrollo, 44(174), 89-117.

Frank, R. H. (1978). Why Women Earn Less: The Theory and Estimation of Differential Overqualification. American Economic Review, 68(3), 360-373.

Freeman, R. B. (1975). The Overeducated American. New York: Academic Press.

Green, D. \& McIntosh, S. (2007). Is There a Genuine Under-utilization of Skills Amongst the Over- qualified. Applied Economics, 39(4), 427-439. doi.org/10.1080/00036840500427 700

Groot, W. \& Van den Brink, H. (2000). Overeducation in the Labor Market: A Meta Analysis, Economics of Education Review, 19(2), 149-158. doi: 10. 1016/S0272-7757(99)000576

Harmon, C. P., Walker, I. \& Nielsen, N. C. W. (2001). Education and earnings in Europe: a cross country analysis of the returns to education. USA: Edward Elgar Publishing. 
Hartog, J. (2000). Over-education and Earnings: Where are We, Where Should We Go. Economics of Education Review, 19(2), 131-147. doi:10.1016/S0272-7757(99)00050-3

Johnson, W. R. (1978). A Theory of Job Shopping, Quarterly Journal of Economics, 92(2), 261-278. doi: $10.2307 / 1884162$

Jovanovic, B. (1979). Firm-specific Capital and Turnover. Journal of Political Economy 87(6), 1246-1260.

Kiker, B. F., Santos, M. C. \& De Oliveira, M. M. (1997) Overeducation and Undereducation: Evidence for Portugal. Economics of Education Review 16(2), 111-112. doi:10.1016/S02727757(96)00040-4

McGuiness, S. (2006). Overeducation in the Labour Market. Journal of Economic Surveys 20(3), 387-418. doi: 10.1111/j.0950-0804.2006.00284.x

Medina, E. (2003). Modelos de elección discreta. Publicaciones Económicas de la Universidad Autónoma de Madrid. Recuperado de: http://www. uam.es/personal_pdi/economicas/ eva/pdf/logit.pdf

Mincer, J. (1974). Schooling, Experience, and Earnings. Human Behavior \& Social Institutions No. 2. Recuperado de: http://eric.ed.gov/?id=ED103621

Mora, J. J. (2005). Sobre-educación en Cali (Colombia), ¿Desequilibrio temporal o permanente?: algunas ideas, 2000-2003, Revista de Economía y Administración, 1(1), 115-144.

Mora, J. J. \& Muro, J. (2008). Sheepskin Effects by Cohorts in Colombia, International Journal of Manpower, 29(2) 111-121. http://dx.doi. org/10.1108/01437720810872686
Ng, Y. C. (2001). Overeducation and Undereducation and their Effect on Earnings: Evidence from Hong Kong, 1986-1996. Pacific Economic Review, 6(3), 401-418. doi: 10.1111/14680106. 00141

Pérez, P. (2005). Sobreeducación en el Mercado de Trabajo argentino en un Período de Desempleo Masivo (1995-2003). Recuperado de: http://www.aset.org.ar/congresos/7/11024.pdf

Psacharopoulos, G. \& Patrinos, H. A. (2004). Returns to investment in education: a further update. $E d u$ cation economics, 12(2), 111-134. doi: 10.1080/0964529042000239 140

Rosen, S. (1972). Learning and Experience in the Labour Market. Journal of Human Resources, 7(3), 326342. doi: 10.2307/145087

Rubb, S. y M. A. Quinn. (2002). Educational Mismatches in Mexico: Additional Evidence of the Importance of Labor Market Assignments? Economics of Education Review, 25(2), 147-156. doi: 10.1016/j.econedurev.2005.01.003

Sicherman, N. (1991). Overeducation in the Labor Market. Journal of Labor Economics, 9(2), 101-122.

Sicherman, N. \& Galor, O. (1990). A theory of career mobility. Journal of Political Economy, 98(1), 169192.

Torres, V. E. \& Celton, D. E. (2009). Discriminación salarial en Argentina entre nativos y paraguayos. Cuadernos geográficos de la Universidad de Granada, 45(2), 263-285. 
Verdugo, R. \& N. Verdugo. (1989). The Impact of Surplus Schooling on Earnings. Journal of Human Resources, 24(4), 629-643. doi: $10.2307 / 145998$
Waisgrais, S. (2005). Determinantes de la Sobreeducación de los Jóvenes en el Mercado Laboral Argentino. En Ponencia presentada en el Séptimo Congreso Nacional de Estudios del Trabajo, Recuperado de: http://www.aset. org.ar/congresos/7/10004.pdf 


\title{
EVOLUTION OF THE CAUSES AND EFFECTS OF EDUCATIONAL DYSFUNCTION IN THE MEXICAN LABOR MARKET 1990-2010'
}

Héctor Alberto Botello Peñaloza ${ }^{2}$

Universidad Industrial de Santander - Article Type 1.

Scientific and Technological Research - Received May 19th 2014 - Accepted: Junio 13th 2015

doi: http://dx.doi.org/10.17981/econcuc.36.1.2015.30

\begin{abstract}
This article studies the causes and effects of educational mismatch in the Mexican labor market, for which population censuses were used from 1990-2010.It was found that the gap is due to a structural problem of the Mexican economy, covering on average $31 \%$ of the employed population, unchanged in the 20 years were studied. The educational gap helps explain part of the wage gap with a positive return for workers with over-education and a penalty in the case of under-education. It was concluded that labor observatories initiatives for graduates to learn the skills and competencies required by employers are a first step to solve the problem of over-education in the country.
\end{abstract}

\section{Keywords:}

Education, human capital, occupational choice, productivity.

JEL: I21, I23, J01, J24

\section{If you to reference this article}

Botello, M. (2015). Evolution of the causes and effects of educational dysfunction in the mexican labor market 1990-2010. Económicas CUC, 36 (1), 275-304. doi: http://dx.doi.org/10.17981/econcuc.36.1.2015.30

\footnotetext{
${ }^{1}$ Research article derived from "settlements and territorial conflicts" Project Regional Development Research Group and Land Planning in the Development Research Line human and conflicts, funded by the Industrial University of Santander.

${ }^{2}$ Professor Industrial University of Santander, Bucaramanga. Colombia. Economist. Master of Industrial Engineering. E-mail: hectoralbertobotello@gmail.com.
} 


\section{INTRODUCTION}

In recent decades, countries have made significant investments in education, seeing reflected in increasing enrollment rates and participation rates in tertiary education. However, part of the knowledge acquired through education may be underutilized if they do not match the skills required by companies. In this sense, the educational mismatch arises when a person is in a job that underestimates or overestimates the human capital endowment that has (Freeman, 1975) there is evidence that both advanced and developing countries have grown different types of educational mismatches. (McGuiness, 2006)

The existence of this phenomenon discourages investment in education, as expected return rates decrease (Mora, 2008). Different explanations have been put forward to explain this phenomenon, such as temporary frictions in the labor market (Johnson, 1978; Sicherman \& Galor, 1990), imperfect information (Jovanovic, 1979), geographical immobility (Frank, 1978), effects signaling and labor mobility (Rosen, 1972; Sicherman \& Galor, 1990; Sicherman, 1991).

According to neoclassical theory, population changes, leading to a disorderly increase in the number of people investing in education, causing that companies can not quickly change their educational requirements, which can lead to a structural imbalance between supply and labor demand. The empirical literature tends to affirm that labor income earned by workers depend on their human capital accumulated as skills developed within the company, Therefore, if a worker is over or under polite will have a penalty against the properly educated (Hartog, 2000). From another point of view, thesignaling models pose this phenomenon as a permanent effect.
Education is a signal used by the workers to display in the labor market that is more suitable and therefore more productive; thus, individuals invest in education to have more opportunities compared to other workers. In this sense, there could be an increase in the number of people with university education and thus saturate the market, causing a widespread problem of education can not be met by the country's productive structure.

However, mismatches can also be positive, given that individuals temporarily performing work for which they are overeducated because they acquire the necessary skills to perform more skilled jobs; for this can be seen that occupational mobility is related to some level of educational mismatch. With this, you can even say that the imbalances would only be a temporary phenomenon in the labor market and not a structural problem between demand and supply of labor (Sicherman, 1991; Kiker, Santos \& De Oliveira, 1997). To empirically study the educational imbalances, it is necessary to take into account the heterogeneity of workers and skills actually acquired by them as a part of the gap may be due to the different skills acquired by individuals with similar levels of education.

In this vein, Chevalier (2003), has spread among individuals apparently over / under-educated, who are similarcompetencies to those of his fellowwork properly educated but with a higher / lower educational level required for the post of job they occupy. On the other hand, workers are truly over-educated / undereducated, suffering an over / under utilization of their skills in the workplace.

Other studies confirm these differences between education and skills mismatch (Di Pietro \& Urwin, 2006; Green \& McIntosh, 2007), highlighting the need to 
consider the heterogeneity of skills between individuals with similar levels of educationand to consider the grade mismatch in skills, in analyzing the economic effects of the mismatch between human capital possessed by individuals and the labor market demand.

In this vein, a systematic review of the empirical literature found you can get the following stylized facts on trends in educational mismatch in the characteristics of workers. (Hartog, 2000; Mora, 2005; Castillo, 2007)

a. The educational mismatch may be a temporary or permanent phenomenon.

b. The rate of return on education over-educated is higher than the under-educated, but lower compar compared to that of adequately educated

c. Over-educated people have less experience than the under-educated.

d. The over-educated tend to easily switch occupation.

e. The over-educated have incomplete and contracts shorter than the under-educated.

f. There are meaningful differences between estimations, depending on the method used.

In this exploration, we can highlight from the empirical point of view, three ways to determine if an individual presents a mismatch in the labor market: the statistical method, the subjective method and objective method.

The statistical method defines the mismatch of an occupational group from the mean plus or minus one standard deviation. An individual is over-educated who have more years of education than the mean plus the standard deviation of the reference group and vice versa for undereducated.

This interpretation implies that a worker can only go from being over or under polite to be properly educated if he changes his occupation, although Dorn \& Souza-Posa (2005), show that it is possible for an individual improves or worsens match play another job within the same occupational group or adjusting the requirements of the current job.

The main drawback of this method is the setting value of reference related to the average educational level of employment to treat. For instance, if a job has a high proportion of over-educated workers, occupational average is increased and therefore, the reference value, so that the level of over-education is underestimated (McGuinness, 2006, 396). This flaw has been exposed mainly by Groot \& van den Brink (2000), in an analysis of 25 occupations, over-education "true" it was $26 \%$, whereas the estimated with the mean and standard deviation gave only $12 \%$. The second problem is the distribution of education levels within the population.

If a systematically repeated number, known as bimodal distribution, the definition of the over-education from the mean plus the standard deviation will measurement errors. To correct the above problems Kiker et al. (1997) for Portugal; Ng (2001), Hong Kong; Rubb \& Quinn (2002) for Mexico, define the educational mismatch from the middle of the wage distribution, which also forms part of the statistical method. However, despite its limitations statistical method is best implemented for ease of calculation and availability statistics. (Hartog, 2000)

The subjective method is to ask the individual what their qualifications in the current job, based on their responses 
indicate if considered over-educated or not. However, subjective measures have misclassification, because the researcher does not know exactly how the worker made inferences about their educational mismatch.

Finally, the objective method requires knowing the skills of individuals and the skills that businesses require. However, it is difficult to know the range of skills of individuals and substitution between them, whet her this method requires some information about the companies.

Empirical researches in Latin America have carried out different exercises in Uruguay (Bucheli \& Casacuberta, 2001; Espino, 2013); Colombia (Caicedo, 2007; Castillo, 2007; Mora, 2008); Mexico (Rubb \& Quinn, 2002) and Argentina (Pérez, 2005; Waisgrais, 2005).

In this vein, for contributing to the international literature on the experience of this phenomenon in Mexico, this paper studies the causes and effects of educational mismatch in the Mexican labor market,understood as the excess or defect difference between the educational attainments of the individual and required by the job in which it operates. For this, the work is organized in four sections including the present. Following the methodology implemented is described, in the third the results are presented and finally presents the conclusions.

\section{METHODOLOGY}

\section{Data source}

In this research, the analysis is based on statistical data from census microdata from population and housing arranged by the Mexican Institute of Statistics (INEGI) and IPUMS project of the University of Minnesota. The total population of the survey is $7,051,284$ people with a total range adjusted expansion factors of 101 million people. Table 1 shows the distribution of census by sex, where men participate with $65 \%$ of employees with a total of 68 million.

Table 1.

Distribution of respondents by sex.

\begin{tabular}{cccc}
\hline Year & Total & Men & Women \\
\hline $\mathbf{1 9 9 0}$ & $18,002,250$ & $13,567,810$ & $4,434,440$ \\
\hline $\mathbf{1 9 9 5}$ & $24,907,861$ & $16,726,047$ & $8,181,814$ \\
\hline $\mathbf{2 0 0 0}$ & $26,089,643$ & $17,493,021$ & $8,596,622$ \\
\hline $\mathbf{2 0 1 0}$ & $32,166,127$ & $20,564,433$ & $11,601,694$ \\
\hline Total & $101,165,881$ & $68,351,311$ & $32,814,570$ \\
\hline
\end{tabular}

Source: EmploymentSurveysMexico1990-2010. INEGI

\section{Model to estimate the causes \\ of educational mismatch}

The phenomenon of study, over or undereducation is empirically estimated as the difference between the educational level of the individualand the mean plus or minus one standard deviation. Determinants models they find that people are in states educational mismatchagainst those who are properly educated are proposed. Statistically these kinds of variables are categorized into each in the event that the person is over-educated / under-educated and zero otherwise.When the analysis variables have these characteristics, have only two values are called dichotomous variables and study discrete choice models are implemented.

In general, it is considered that behind the dependent variable in these models, $\mathrm{Y}$, is an unobservable variable, I, that depends on a set of explanatory variables $\mathrm{X} \_\mathrm{i}$ takes certain values if you have exceeded certain threshold, as expressed in below. 


$$
Y_{i}=\left\{\begin{array}{l}
1 \text { if } I_{i}^{*}>0 \text { this is when } X_{i} \beta+\mathcal{E}_{i}>0 \\
0 \text { if } I_{i}^{*}<0 \text { this is when } X_{i} \beta+\mathcal{E}_{i}<0
\end{array}\right.
$$

Where the assumption on the distribution of $\varepsilon$, determines the type of model to estimate: if a function of uniform distribution is assumed, the truncated linear probability model is used; if it is normally distributed with zero mean and unit variance, the generated model will be a probit; while if it is supposed to be using a logistic curve, it would be a logit model. The hypothesis that the threshold to overcome the latent variable is zero, can be modified by any other suggesting that the critical value is the value defined by the constant term (Pérez, 2005). Under the first approach the probabilistic model would be defined (Medina, 2003)

$$
\begin{aligned}
& P_{i}=\operatorname{Pr} o b\left(Y_{i}=1\right)=\operatorname{Pr} o b\left(I_{i}^{*}>0\right)= \\
& \operatorname{Pr} o b\left(X_{i} \beta+\mathcal{E}_{i}>0\right)=F\left(X_{i} \beta\right)
\end{aligned}
$$

The effectiveness of these models is measured through two indicators: the first is the degree of the variance of the dependent variable captured or predicted by the independent variables, indicator called R2. The highest is the most effective model to determine the behavior of the dependent variable and vice versa. The second is the degree of observations of the model has been classified correctly in the analyzed characteristic, a higher percentage indicates the degree of robustness of the model is better. In statistical terms, there are different situations on the nature of the data that must be corrected for optimal calibration of the model such as the presence of heteroscedasticity, multicollinearity and serial autocorrelation (Pérez, 2005). Taking into account the above criteria, this paper proposes to use probabilistic function as follows:

$$
\begin{aligned}
& Y=f(S, X) \\
& Y=a+\beta_{i} X_{i}+\mathcal{E}
\end{aligned}
$$

Where $\mathrm{Y}$ is the dependent variable to be explained or categorized dichotomously, where 1 is when the person is over-educated / under-educated and zero otherwise. Meanwhile, 8 , capture the marginal contribution of each of these factors to the probability of being over-educated / under-educated, while $\varepsilon$ is the error of the estimate is distributed according to the probability function. The latter is explained by a table summarizing factors characteristic of the person, $\mathrm{X}$, which were selected based on the assumptions:

H1: Being a woman I increase the probability of being under-educated.(Hartog, 2000; Mora, 2005; Castillo, 2007)

H2: Individuals who have higher education degrees tend to be over-educated while generally have less experience. (Hartog, 2000)

\section{Model to estimate the effect of the gap in earnings}

To evaluate the effect of educational mismatch in the labor market, labor income is estimated hourly different types of workers, using the model of Mincer (1974). This specification involves the characteristics of workers as determinants of the earnings of the employed, it is one of themodels most explored and known in the literature on labor economics. (Psacharopoulos, 2004; Cohn \& Addison, 1998; Asplund \& Pereira, 1999; Harmon, Walker \& Nielsen, 2001). This model of a semilogarithmic function (Equation 1) estimated by the method of ordinary least squares (OLS), having as dependent variable the logarithm of wages of workers and as independent variables the years of education of the worker, work experience (and its square), the branch of activity, occupation and possession of contract.

$$
\begin{aligned}
\log (\mathrm{Y})= & B_{0}+B_{1} \operatorname{Educ}+B_{2} \operatorname{Exp}+ \\
& B_{3} \operatorname{Exp} 2+B_{4} \mathrm{~S}+B_{5} \mathrm{~L}+\varepsilon
\end{aligned}
$$


- $Y$ is the hourly labor income of the individual

- Educ is the educational level.

- Expare the years of work experience

- Lcorresponds to educational mismatch presented by the worker.

- $\varepsilon$ is a random disturbance term that is normally distributed

Among the other variables of the worker to be controlled in this study, there is the province where the individual lives, marital status, ethnicity, among others. In terms of interpretation, the coefficients (B) represent the percentage change of labor income will have on each of the changes on the characteristics of workers. The effectiveness of this model is measured by the variance of the dependent variable captured by the control variables. This indicator is called $\mathrm{R} 2$. If this is higher, is more effective the model to determine the behavior of the dependent variable. There are different situations on the nature of the data must also arrange for proper calibration of the model such as the presence of heteroscedasticity, multicollinearity and serial autocorrelation.

We must also fix another problem that is implicit in the data, the so-called selection bias. What is the lack of sampling randomness, or non-random sample selection in household surveys carried out (De Oliveira and Ariza, 2000). It called bias, because a sample that is concentrated in certain population group diverts the result of a variable of interest in the group with greater representation. This problem is necessary to correct when it investigates differences in earnings of people on specific population groups, it does not allow accurate results of the phenomenon analyzed.

Heckman correction is the solution most often used in the literature to estimate effects (Heckman, 1979; De Oliveira \&Ariza, 2000). It develops, estimating two re- gressions, the first is done on the basis of the participation equation, defined as the probability that the individual participates in the labor market against the factors that can influence the choice.

$$
\mathrm{pi}=B o+z i \varphi+u i
$$

Where pi refers to the probability of participating in the labor market, zi corresponds to a table with explanatory variables that influence the decision to participate, $\varphi$ is a vector of parameters, and ui are errors. After done, the second calculation is the Mincer equation incorporating the correction selection by lambda $(\lambda)$ as the inverse ratio of Mill (De Oliveira and Ariza, 2000)

$$
\begin{array}{ll}
\log (\mathrm{Y})= & \text { B0+B1S + B2Exp }+ \\
& \text { B3Exp2 }+B 4 \mathrm{~L}+\lambda \mathrm{ti} \theta+\varepsilon \mathrm{i}
\end{array}
$$

This has corrected the selection bias. Using the argument of Torres \& Celton (2009), the importance of using $(\lambda)$ is determined by estimating the regressionprovidedby itssignificant coefficient. In the case of not being representative, one could work directly without the need for correction. For this type of calculation you want to inquire about the assumptions offered in literature:

H3: The over-educated earn more than infraeducated, but earn less than those who are adequately educated (Verdugo and Verdugo, 1989). However, it has also been found that there are positivereturns to over-education and negative to the infra-education (Cohn \& Kahn, 1995; Duncan \& Hoffman, 1981).

H4: There are significant differences between the returns to over-educated in different occupations. Verdugo \& Verdugo (1988); Cohn \& Kahn (1995) and Kiker, Santos \& Oliveira (1997)

\section{RESULTS}

Table 2 presents the descriptive statistics regarding the distribution of workers in total employment base; it found that 
the degree of job mismatch affects $31 \%$ of employed Mexicans, with 16\% overeducated and under-educated 15\%. Gender mismatch affects slightly more men $32 \%$ vs. women with $29.6 \%$. By economic sector, $40 \%$ of primary workers presents educational mismatch against 30\% secondary and tertiary (29\%). Likewise, workers with greater human capital endowment (approximately age or educational level), often suffer the mismatch, especially giftedness,for example $40 \%$ of workers with university degrees appear to be over-educated for positions against $6.3 \%$ of primary, evidence which is common for developing countries, where lack of job opportunities in sectors that demand for workers with high human capital endowment is common. (Rubb \& Quinn, 2002)
The inter-temporal monitoring of educational mismatch exposed in Figure 1 of the Mexican labor market indicates that they have not been significant changes between 1990 and 2010 remains around 31\%. However, there is a slight reduction in the levels of under-educated (-2\%) and an increase of educated $(+2 \%)$.

On the model to estimate the causes of educational mismatch 594.972 individuals were selected for regression with undereducated and 613,000 individuals for overeducated. Subsequently the chi-square tests were performed to test the function type with which the dependent variable was distributed, finding that for both exercises was by a representation of a logistic curve, however, it was also decided to construct the model assuming distribution type probit.

Table 2.

Percentage of workers by type of job mismatch. Descriptive statistics. 2010

\begin{tabular}{|c|c|c|c|c|c|}
\hline \multicolumn{2}{|c|}{ Variable } & Mismatch & Under-educated & Over-educated & correctly \\
\hline \multicolumn{2}{|r|}{ Total } & 31.0 & 14.8 & 16.2 & 69.0 \\
\hline \multirow{2}{*}{ Sex } & Man & 31.8 & 14.7 & 68.2 & 17.1 \\
\hline & Woman & 29.6 & 15.1 & 70.4 & 14.5 \\
\hline \multirow{3}{*}{ Sector } & Primary & 40.4 & 20.0 & 59.6 & 20.4 \\
\hline & Secondary & 30.1 & 14.8 & 69.9 & 15.3 \\
\hline & Tertiary & 29.1 & 13.6 & 70.9 & 15.5 \\
\hline \multirow{5}{*}{ Age } & Less than 25 & 26.9 & 6.9 & 73.1 & 20.0 \\
\hline & 25 to 35 & 27.7 & 8.9 & 72.3 & 18.8 \\
\hline & 35 to 45 & 28.3 & 12.4 & 71.7 & 15.9 \\
\hline & 45 to 55 & 34.5 & 21.2 & 65.5 & 13.3 \\
\hline & 55 or more & 47.1 & 39.6 & 52.9 & 7.5 \\
\hline \multirow{3}{*}{ Education } & Primary & 13.1 & 6.8 & 86.9 & 6.3 \\
\hline & High School & 43.0 & 3.5 & 57.0 & 39.5 \\
\hline & academic & 40.7 & 0.4 & 59.3 & 40.3 \\
\hline \multirow{4}{*}{ Area } & Up to 2,499 people & 34.8 & 19.8 & 65.2 & 15.0 \\
\hline & 2,500 to 14,999 & 31.9 & 16.7 & 68.1 & 15.2 \\
\hline & 15,000 to 99,999 & 30.6 & 14.5 & 69.4 & 16.1 \\
\hline & 100,000 or more & 29.4 & 12.4 & 70.6 & 16.9 \\
\hline \multicolumn{2}{|c|}{ expanded comments } & $11,929,770$ & $5,703,465$ & $6,226,305$ & $26,556,653$ \\
\hline
\end{tabular}

Source: author's calculations based on population censuses Mexico 2010. INEGI 


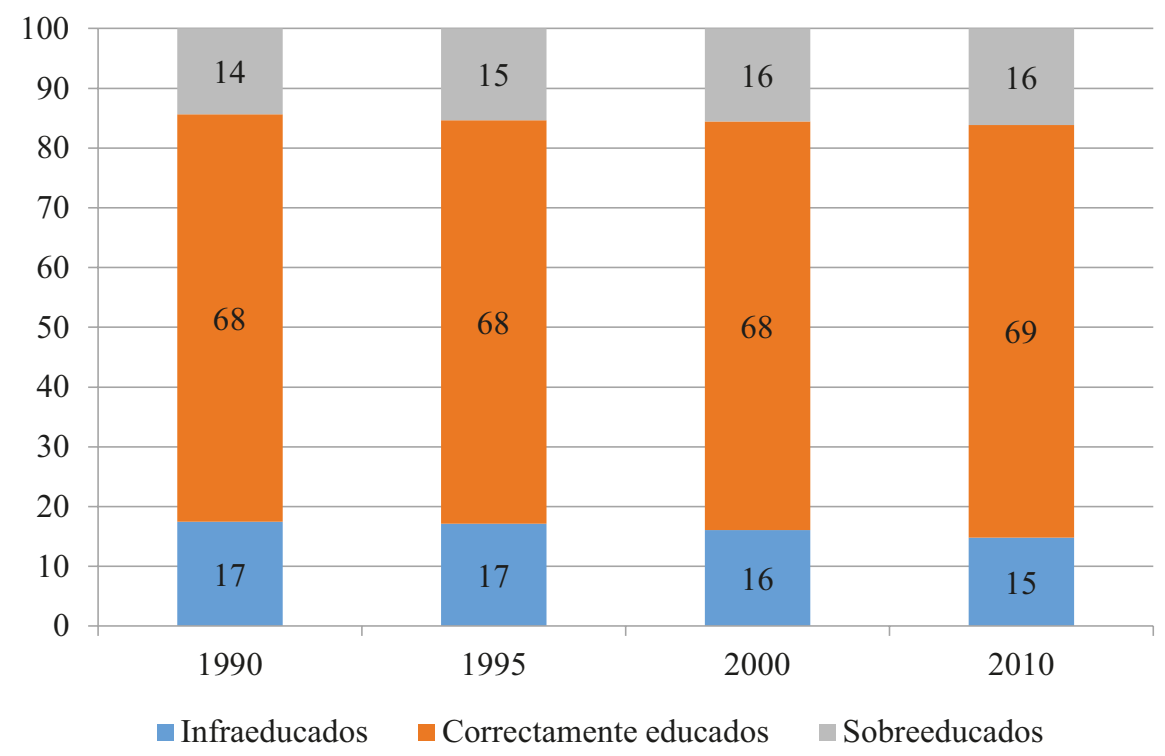

Figure 1. Percentage of educational mismatch by type and year. 1990-1995-2000-2010

Source: Own development based on population censuses Mexico1990-2010. INEGI

In this vein, Table 3 shows the estimation results of logit models with coefficients and elasticities (marginal effect) of each of thecontrol variables (independent) over the likelihood of the individual to be over-educated or infra-educated between 1990 and 2010 .

In terms of overall fit, the model is acceptable considering that the statistical valuechi 2 is very significant being the probability that the linear combination model is not significant zero.In turn, all input variables presented statistical significance level of $5 \%$ based on the adjusted R2, the models fail to explain between $38.5 \%$ and $39.3 \%$ of the variance of the dependent variables,while the percentage of cases correctly classified within the proposed model reached about $85 \%$. With this verification of robustness of the model is passed to check each of the hypotheses proposed for the work.

For the interpretation of the results, the marginal effect (elasticity) for continuous variables, equal to the percentage by which the probability of being over-educated/infra-educated taken a $1 \%$ change in the independent variable of analysis increases.For categorical variables, the marginal effect measures the probability of a relative manner as compared to a base feature.In the initial hypothesis of the literature, are evidence that being a woman increases the probability of being under-educated (Hartog, 2000; Mora, 2005; Castillo, 2007), the Mexican has a $2.5 \%$ more likely to be under-educated in their occupation in relation to men.

In terms of education, it appears that individuals who have higher human capital endowments tend to be overeducated (Hartog, 2000), but less to be under-educated.For example, workers with graduate have a $19 \%$ chance of being overqualified that they do not have any education, also they have $3.8 \%$ less likely to be under-educated.In terms of experience, over-educated workers, despite being better educated, they are often less experienced, in fact an additional year of age decreases the probability of being over-qualified $-0.2 \%$. 
Table 3 .

Logit model estimates of probability of being under-educated or over-educated

\begin{tabular}{|c|c|c|c|c|c|c|c|c|}
\hline \multirow[b]{2}{*}{ Variable } & \multirow{2}{*}{$\begin{array}{c}\text { Feature } \\
\text { Evaluated }\end{array}$} & \multicolumn{3}{|c|}{ Over-educated } & \multicolumn{3}{|c|}{ Under-educated } & \multirow[b]{2}{*}{$\begin{array}{l}\text { Feature } \\
\text { Base }\end{array}$} \\
\hline & & $\begin{array}{l}\text { Marginal } \\
\text { effect }\end{array}$ & Error & $\mathrm{p}(\mathrm{z})$ & $\begin{array}{l}\text { Marginal } \\
\text { effect }\end{array}$ & Error & $\mathrm{p}(\mathrm{z})$ & \\
\hline \multicolumn{2}{|c|}{ Age (proxy experience) } & $-0.03 \%$ & 0.0000 & 0 & $0.1 \%$ & 0.0000 & 0 & Continuing \\
\hline $\begin{array}{c}\text { Head of } \\
\text { household }\end{array}$ & Yes & $-1.4 \%$ & 0.0010 & 0 & $2.3 \%$ & 0.0009 & 0 & No \\
\hline Sex & Woman & $-1.3 \%$ & 0.0009 & 0 & $2.5 \%$ & 0.0010 & 0 & Man \\
\hline \multirow{3}{*}{$\begin{array}{l}\text { Education } \\
\text { level }\end{array}$} & Primary & $5.0 \%$ & 0.0168 & 0 & $-0.6 \%$ & 0.0014 & 0 & \multirow{3}{*}{ none } \\
\hline & High School & $9.5 \%$ & 0.0009 & 0 & $-4.3 \%$ & 0.0008 & 0 & \\
\hline & Academic & $19.0 \%$ & 0.0012 & 0 & $-3.8 \%$ & 0.0007 & 0 & \\
\hline \multirow{8}{*}{ Occupation } & Professional & $-1.6 \%$ & 0.0016 & 0 & $1.8 \%$ & 0.0039 & 0 & \multirow{8}{*}{ Directors } \\
\hline & Technicians & $10.1 \%$ & 0.0065 & 0 & $-5.3 \%$ & 0.0017 & 0 & \\
\hline & Office Workers & $19.1 \%$ & 0.0092 & 0 & $-7.0 \%$ & 0.0012 & 0 & \\
\hline & $\begin{array}{l}\text { Service workers } \\
\text { and salesmen }\end{array}$ & $48.6 \%$ & 0.0132 & 0 & $-14.5 \%$ & 0.0011 & 0 & \\
\hline & $\begin{array}{c}\text { Skilled } \\
\text { agricultural } \\
\text { workers } \\
\end{array}$ & $71.8 \%$ & 0.0122 & 0 & $-22.6 \%$ & 0.0021 & 0 & \\
\hline & $\begin{array}{l}\text { Industry } \\
\text { operators }\end{array}$ & $61.5 \%$ & 0.0128 & 0 & $-16.1 \%$ & 0.0011 & 0 & \\
\hline & $\begin{array}{c}\text { Machine } \\
\text { operators and } \\
\text { assemblers }\end{array}$ & $65.4 \%$ & 0.0118 & 0 & $-11.1 \%$ & 0.0008 & 0 & \\
\hline & $\begin{array}{c}\text { Unskilled } \\
\text { workers }\end{array}$ & $77.1 \%$ & 0.0097 & 0 & $-15.4 \%$ & 0.0010 & 0 & \\
\hline \multirow{2}{*}{ Branch } & Industry & $-3.0 \%$ & 0.0019 & 0 & $4.5 \%$ & 0.0027 & 0 & \multirow{2}{*}{ agriculture } \\
\hline & Services & $-5.2 \%$ & 0.0030 & 0 & $5.7 \%$ & 0.0023 & 0 & \\
\hline \multirow{6}{*}{ Year } & 1995 & $0.4 \%$ & 0.0024 & 0.1 & $1.1 \%$ & 0.0035 & 0.002 & \multirow{3}{*}{1990} \\
\hline & 2000 & $-2.1 \%$ & 0.0013 & 0 & $3.7 \%$ & 0.0011 & 0 & \\
\hline & 2010 & $-4.7 \%$ & 0.0026 & 0 & $8.4 \%$ & 0.0012 & 0 & \\
\hline & Observations & \multicolumn{2}{|c|}{594,972} & & \multicolumn{2}{|c|}{613,000} & & \\
\hline & $\mathrm{R} 2$ & \multicolumn{2}{|c|}{0.385} & & \multicolumn{2}{|c|}{0.393} & & \\
\hline & $\begin{array}{c}\text { Correct } \\
\text { classification }\end{array}$ & \multicolumn{2}{|c|}{$87.6 \%$} & & \multicolumn{2}{|c|}{$84.0 \%$} & & \\
\hline
\end{tabular}

Source: author's calculations based on population censuses Mexico 1990-2010. INEGI

This can confirm that Mexicans with high educational levels choose jobs that allow them to gain experience and then get better jobs. Other interesting results obtained in the exercise are decreasing year by year in the probability of being over-educated to be increased under-educated.

In this sense, workers in 2010 had a $-4.7 \%$ being over-educated than those in 1990 and $8.4 \%$ more in being under-educated. Then he proceeded to make the Mincer 
equation Heckman correction for the effect of educational mismatch in earnings per hour for each of the census years available (1990-1995-2000-2010), whose results are shown in table 4.The total number of workers was 99.2 million with an average degree of variance of $35 \%$. The model is logarithmic so the change of $1 \%$ in continuous variables is interpreted as the percentage change in the dependent variable.

Similarly categorical variables indicate a percentage change relative to a base feature. It is appreciated that there is statistical evidence supporting that there are positivereturns to over-education and negative to the infra-education (Cohn \& Kahn, 1995; Duncan \& Hoffman, 1981). On average the under-educated earn $4.95 \%$ less than properly trained while over-educated earn $9.1 \%$ more. A significantly volatile effect in the year 1995 is seen when the gain of the properly educated increases to $11 \%$ and the over-educated $24 \%$.

These results may be due to the economic crisis suffered by Mexico because of the te- quila crisis this year, forcing a drop in wages. This movement, more adversely affect the low-income widening wage gap between groups.

This shows that the educational mismatch is a phenomenon that can be affected by the economic cycle, something that has not been studied in detail in the literature. Table 4 exercises on estimates of returns by type of occupation are showed. Significant differences between the returns to overeducated in different occupations are found as in Verdugo \& Verdugo (1988); Cohn \& Kahn (1995) and Kiker, Santos \& Oliveira (1997).

Also, we see that are in occupations where higher human capitalis required where the biggest differences are observed. For example, properly educated professionals earn about of $22 \%$ than the under-educated and over-educated $27.7 \%$. In non-university technicians differential is $11.6 \%$ and $20.3 \%$ respectively. Meanwhile, office workers, machine operators and industrial not have a high differential (see Table 5).

Table 4.

Result estimates of labor income per hour by Heckman correction. Years 1990 - 1995 - 2000 - 2010

\begin{tabular}{|c|c|c|c|c|c|c|}
\hline Variable & $\begin{array}{c}\text { Characteristic } \\
\text { Evaluated }\end{array}$ & 1990 & 1995 & 2000 & 2010 & $\begin{array}{c}\text { Characteristic } \\
\text { base }\end{array}$ \\
\hline \multirow{2}{*}{ Mismatch } & properly educated & $3.0 \%$ & $11.4 \%$ & $4.0 \%$ & $1.4 \%$ & \multirow{2}{*}{ Under-educated } \\
\hline & Over-educated & $5.9 \%$ & $24.4 \%$ & $4.6 \%$ & $1.5 \%$ & \\
\hline $\begin{array}{c}\text { Head of } \\
\text { household }\end{array}$ & Yes & $8.7 \%$ & $7.6 \%$ & $8.5 \%$ & $6.7 \%$ & No \\
\hline Sex & Woman & $-8.6 \%$ & $-4.4 \%$ & $-7.3 \%$ & $-7.5 \%$ & Man \\
\hline \multirow{4}{*}{ Age } & 25 to 35 & $14.2 \%$ & $20.0 \%$ & $15.7 \%$ & $12.6 \%$ & \multirow{4}{*}{ Under 25} \\
\hline & 36 to 45 & $20.3 \%$ & $22.8 \%$ & $21.1 \%$ & $17.8 \%$ & \\
\hline & 46 to 55 & $20.3 \%$ & $23.1 \%$ & $20.6 \%$ & $18.7 \%$ & \\
\hline & 56 onwards & $9.7 \%$ & $10.1 \%$ & $10.4 \%$ & $10.1 \%$ & \\
\hline \multirow{3}{*}{ Education } & Primary & $15.8 \%$ & $14.6 \%$ & $15.0 \%$ & $16.6 \%$ & \multirow{3}{*}{ None } \\
\hline & High School & $35.7 \%$ & $26.3 \%$ & $44.0 \%$ & $40.2 \%$ & \\
\hline & Academic & $59.7 \%$ & $37.4 \%$ & $75.3 \%$ & $70.2 \%$ & \\
\hline
\end{tabular}


Cont...

\begin{tabular}{|c|c|c|c|c|c|c|}
\hline Variable & $\begin{array}{c}\text { Characteristic } \\
\text { Evaluated }\end{array}$ & 1990 & 1995 & 2000 & 2010 & $\begin{array}{c}\text { Characteristic } \\
\text { base }\end{array}$ \\
\hline Immigrant & Yes & $28.5 \%$ & $40.2 \%$ & $31.8 \%$ & $24.9 \%$ & No \\
\hline \multirow{11}{*}{$\begin{array}{l}\text { Branch of } \\
\text { activity }\end{array}$} & mining & $43.3 \%$ & $44.4 \%$ & $27.4 \%$ & $39.6 \%$ & \multirow{11}{*}{ Agriculture } \\
\hline & Industry & $18.6 \%$ & $13.0 \%$ & $9.6 \%$ & $1.2 \%$ & \\
\hline & $\begin{array}{c}\text { Electricity, gas and } \\
\text { water }\end{array}$ & $34.7 \%$ & $36.3 \%$ & $37.4 \%$ & $33.1 \%$ & \\
\hline & Building & $21.3 \%$ & $13.2 \%$ & $14.5 \%$ & $17.4 \%$ & \\
\hline & Commerce & $19.3 \%$ & $16.2 \%$ & $5.1 \%$ & $1.9 \%$ & \\
\hline & $\begin{array}{l}\text { Hotels and } \\
\text { restaurants }\end{array}$ & $14.1 \%$ & $13.4 \%$ & $4.4 \%$ & $7.5 \%$ & \\
\hline & $\begin{array}{l}\text { Transport and } \\
\text { communications }\end{array}$ & $29.5 \%$ & $18.6 \%$ & $12.1 \%$ & $4.0 \%$ & \\
\hline & $\begin{array}{c}\text { Financial services } \\
\text { and insurance }\end{array}$ & $40.5 \%$ & $43.5 \%$ & $36.5 \%$ & $27.3 \%$ & \\
\hline & $\begin{array}{c}\text { Public administration } \\
\text { and defense }\end{array}$ & $11.0 \%$ & $32.7 \%$ & $14.9 \%$ & $19.2 \%$ & \\
\hline & Education & $30.8 \%$ & $41.3 \%$ & $36.7 \%$ & $42.5 \%$ & \\
\hline & Other services & $18.7 \%$ & $16.0 \%$ & $6.2 \%$ & $3.5 \%$ & \\
\hline \multirow{8}{*}{ Occupation } & Professional & $-17.2 \%$ & $25.4 \%$ & $-18.9 \%$ & $-6.3 \%$ & \multirow{8}{*}{ Directors } \\
\hline & $\begin{array}{l}\text { Technical non- } \\
\text { university }\end{array}$ & $-41.7 \%$ & $-14.7 \%$ & $-37.9 \%$ & $-16.6 \%$ & \\
\hline & Office Workers & $-46.6 \%$ & $-28.1 \%$ & $-47.9 \%$ & $-38.7 \%$ & \\
\hline & Salesmen & $-65.7 \%$ & $-58.4 \%$ & $-69.6 \%$ & $-51.3 \%$ & \\
\hline & agricultural workers & $-86.3 \%$ & $-81.1 \%$ & $-79.1 \%$ & $-58.1 \%$ & \\
\hline & Industry operators & $-57.9 \%$ & $-45.1 \%$ & $-63.6 \%$ & $-37.4 \%$ & \\
\hline & Machine operators & $-62.0 \%$ & $-44.0 \%$ & $-64.1 \%$ & $-43.7 \%$ & \\
\hline & Unskilled workers & $-73.1 \%$ & $-53.1 \%$ & $-72.2 \%$ & $-51.8 \%$ & \\
\hline \multirow{3}{*}{ population } & $\begin{array}{c}2500 \text { to } 14999 \\
\text { inhabitants }\end{array}$ & $14.7 \%$ & $6.3 \%$ & $5.3 \%$ & $5.1 \%$ & $\begin{array}{c}1 \text { to } 2,499 \\
\text { inhabitants }\end{array}$ \\
\hline & 15000 to 99999 & $17.8 \%$ & $10.3 \%$ & $7.9 \%$ & $8.2 \%$ & \\
\hline & 100.000 or more & $21.5 \%$ & $22.5 \%$ & $16.9 \%$ & $15.0 \%$ & \\
\hline \multirow{3}{*}{$\begin{array}{l}\text { Class of } \\
\text { worker }\end{array}$} & itself & $-31.7 \%$ & $-41.0 \%$ & $-34.0 \%$ & $-26.4 \%$ & \multirow{3}{*}{ employer } \\
\hline & Employee or laborer & $-46.2 \%$ & $-54.0 \%$ & $-50.2 \%$ & $-36.5 \%$ & \\
\hline & Day laborer & $-29.7 \%$ & $-34.6 \%$ & $-50.9 \%$ & $-32.4 \%$ & \\
\hline \multicolumn{2}{|c|}{ Constant } & 8.2324 & 1.8778 & 3.0505 & 3.4221 & \\
\hline
\end{tabular}

\begin{tabular}{ccccc}
\hline Observations & $17,642,390$ & $24,389,234$ & $25,645,373$ & $31,549,220$ \\
\hline R2 & 0.2343 & 0.4053 & 0.4273 & 0.3302 \\
\hline
\end{tabular}

Source: author's calculations based on population censuses Mexico1990-2010. INEGI 
Table 5.

Gains hourly labor income of under-educatedby occupation. Years 1990-2010

\begin{tabular}{ccc}
\hline Occupation & Properly & Overeducated \\
\hline Professional & $22.64 \%$ & $27.73 \%$ \\
\hline $\begin{array}{c}\text { Technical non- } \\
\text { university }\end{array}$ & $11.68 \%$ & $20.35 \%$ \\
\hline Office workers & $0.29 \%$ & $4.89 \%$ \\
\hline Salesmen & $6.77 \%$ & $10.08 \%$ \\
\hline $\begin{array}{c}\text { agricultural } \\
\text { workers }\end{array}$ & $10.69 \%$ & $16.57 \%$ \\
\hline $\begin{array}{c}\text { Industry } \\
\text { operators }\end{array}$ & $0.97 \%$ & $2.41 \%$ \\
\hline $\begin{array}{c}\text { Machine } \\
\text { operators }\end{array}$ & $0.80 \%$ & $2.10 \%$ \\
\hline $\begin{array}{c}\text { Unskilled } \\
\text { workers }\end{array}$ & $5.86 \%$ & $9.68 \%$ \\
\hline
\end{tabular}

Source: author's calculations based on population censuses Mexico1990-2010. INEGI

Among other interesting results it shows that women earn on average $7 \%$ less than men because of gender wage discrimination that exists in Mexico (De Oliveira \&Ariza, 2000). In terms of age there is a growinggain as it grows as it relates to the above with greater informal human capital, in fact on average people of 36-55 years old have $20 \%$ higher salaries than their counterparts under 25 years. However, from this age the return decreases to $10 \%$ which shows that the function relationship between age and wages is concave and negative over time (Mincer, 1974).Meanwhile, the heads of household earning 7\% more than those who are not.Those employed in the tertiary sector also obtained $30 \%$ that of the primary sector while the secondary $25 \%$.

\section{CONCLUSIONS}

The presence of educational mismatches in the labor market, is a sign of concern for agents, as this discourages investment in education since the expected return rates decrease (Mora, 2008).Therefore, the importance in developing countries achieves parity between the posts on offer and the demand for workers.In this sense, this study showed the presence of structural educational mismatches in the Mexican labor market close to $31 \%$ where the over-education is majority with $16.2 \%$ and the under-education $14.8 \%$ that have not submitted changes in 20 years of study.

The results show that there are gender differences in the over-education. In terms of labor mobility, over-educated have less experience than the under-educated and the probability of obtaining a temporary contract is greater.The theory of competition for jobs is applied in the case of workers with less human capital, who are employed in order to obtain a contract, regardless of the educational mismatch. In estimating the returns associated with hourly labor income and type of educational mismatch, it is observed that the latter helps explain part of the observed wage gap between workers occupying a similar position, with a positive return for the years of over- education and a penalty on wages in the case of under-education.

However, differences between different occupations are observed, with those who need more skills where higher penalties are presented by type of mismatch.The results also show that the over-educated singles temporary jobs where they are over-educated becausethey want to acquire skills to work in other areas.But the existence of overeducation in all levels of education opened the discussion about the skills acquired in educational institutions and skills that are needed in the labor market.

This asymmetry should lead countries to adopt a policy of improving the skills of the workforce changing educational systems. The first step is to understand these differences and then review the programs of educational institutions to make them more relevant each time.It is in this sense that labor observatories initiatives for graduates to learn the skills of graduates and those required by employers are a first step to solve the problem of over-education in the country. 
Among the study's limitations inability to study the mismatch was found from a structural point of viewsince in the database noowned implicit needs of businesses.The results of these exercises could confirm that not only the years of education but also the skills acquired by workers actually affect the determination of wages.Finally it was found that the statistical method, despite its limitations, gives results consistent with the theory.

\section{REFERECES}

Asplund, R. \& Telhado, P. (1999). Returns to Human Capital in Europe. Retrieved from: http://www.etla.fi/wp-content/uploads/b156.pdf

Bucheli, F. \& Casacuberta, C. (2001). Sobreeducación y prima salarial de los trabajadores con estudios universitarios en Uruguay. Retrieved from: http:// www.fcs.edu.uy/archivos/Doc0601.pdf

Castillo, M. (2007). Desajuste educativo por regiones en Colombia: ¿competencia por salarios o por puestos de trabajo? Cuadernos de Economía, 26(46), 108 145 .

Chevalier, A. (2003). Measuring Overeducation. Economica, 70(279), 509-531. doi: 10.1111/1468-0335.t01-1-00296

Chon, E. \& Addison, J. (1998). The Economics Returns to Lifelong Learning in OECD Countries. Education Economics, 6(3), 253-307. doi: $10.1080 / 09645299800000021$

Cohn, E. \& S. Khan. (1995). The Wage Effects of Overschooling Revisited. Labour Economics, 2(1), 67-76. doi: 10.1016/09275371(95)80008-L

De Oliveira, O. \& Ariza, M. (2000). Género, trabajo y exclusión social en México. Estudios demográficos y urbanos, 15(43), 11-33.

Di Pietro, G. \& Urwin, P. (2006). Education and Skills Mismatch in the
Italian Graduate Labor Market.Applied Economics, 38(1), 79-93. doi: 10.1080/00036840500215303

Dorn, D. \& A. Souza-Poza. (2005). Overqualification: Permanent or Transitory, paper presented at the International Conference on Educational Economics. Retrieved from: http://citeseerx.ist.psu. edu/viewdoc/download?doi=10.1.1.172.8623 $\&$ rep $=$ rep $1 \&$ type $=p$

Duncan, G. \& Hoffman, S. D. (1981). The Incidence and Wage Effects of Overeducation. Economics of Education Review, 1(1), 75-86. doi: 10.1016/02727757(81)90028-5

Durán, J. \& J. J. Mora. (2006). Una aproximación empírica a la relación entre el desempleo y las vacantes para Popayán, 2001-2005. Lecturas de Economía, 65(2), 209-222.

Espino, A. (2013). Brechas salariales en Uruguay: género, segregación y desajustes por calificación. Problemas del desarrollo, 44(174), 89-117.

Frank, R. H. (1978). Why Women Earn Less: The Theory and Estimation of Differential Overqualification. American Economic Review, 68(3), 360-373.

Freeman, R. B. (1975). The Overeducated American. New York: Academic Press.

Green, D. \& McIntosh, S. (2007). Is There a Genuine Under-utilization of Skills Amongst the Over-qualified. Applied Economics, 39(4), 427-439. doi. org/10.1080/00036840500427700

Groot, W. \& Van den Brink, H. (2000). Overeducation in the Labor Market: A Meta Analysis, Economics of Education Review, 19(2), 149-158. doi: 10.1016/S0272-7757(99)00057-6

Harmon, C. P., Walker, I. \& Nielsen, N. C. W. (2001). Education and earnings in Europe: a cross country analysis of the returns to education. USA: Edward Elgar Publishing. 
Hartog, J. (2000). Over-education and Earnings: Where are We, Where Should We Go. Economics of Education Review, 19(2), 131-147. doi: 10.1016/ S02727757(99)00050-3

Johnson, W. R. (1978). A Theory of Job Shopping, Quarterly Journal of Economics, 92(2), 261-278. doi: 10.2307/1884162

Jovanovic, B. (1979). Firm-specific Capital and Turnover. Journal of Political Economy, 87(6), 1246-1260.

Kiker, B. F., Santos, M. C. \& De Oliveira, M. M. (1997) Overeducation and Undereducation: Evidence for Portugal. Economics of Education Review, 16(2), 111-112. doi: 10.1016/ S02727757(96)00040-4

McGuiness, S. (2006). Overeducation in the Labour Market. Journal of Economic Surveys, 20(3), 387-418. doi: 10.1111/j.0950-0804.2006.00284.x

Medina, E. (2003). Modelos de elección discreta. Publicaciones Económicas de la Universidad Autónoma de Madrid. Retrieved from: http://www.uam.es/personal_pdi/economicas/eva/pdf/logit.pdf

Mincer, J. (1974). Schooling, Experience, and Earnings. Human Behavior \& Social Institutions No. 2. Retrived from: http://eric.ed.gov/?id=ED103621

Mora, J. J. (2005). Sobre-educación en Cali (Colombia), ¿Desequilibrio temporal o permanente?: algunas ideas, 20002003. Revista de Economía y Administración, 1(1), 115-144.

Mora, J. J. \& Muro, J. (2008). Sheepskin Effects by Cohorts in Colombia. International Journal of Manpower, 29(2) 111-121. http://dx.doi.org/ 10.1108/01437720810872686

Ng, Y. C. (2001). Overeducation and Undereducation and their Effect on Earnings: Evidence from Hong Kong, 19861996. Pacific Economic Review, 6(3), 401-418. doi: 10.1111/1468-0106.00141
Pérez, P. (2005). Sobreeducación en el Mercado de Trabajo argentino en un Período de Desempleo Masivo (19952003). Retrived from: http://www. aset.org.ar/congresos/7/11024.pdf

Psacharopoulos, G. \& Patrinos, H. A. (2004). Returns to investment in education: a further update. Education economics, 12(2), 111-134. doi: 10.1080/0964529042000239140

Rosen, S. (1972). Learning and Experience in the LabourMarket. Journal of Human Resources, 7(3), 326-342. doi: $10.2307 / 145087$

Rubb, S. y M. A. Quinn. (2002). Educational Mismatches in Mexico: Additional Evidence of the Importance of Labor Market Assignments? Economics of Education Review, 25(2), 147-156. doi: 10.1016/j.econedurev.2005.01.003

Sicherman, N. (1991). Overeducation in the Labor Market. Journal of Labor Economics, 9(2), 101-122.

Sicherman, N. \& Galor, O. (1990). A theory of career mobility. Journal of Political Economy, 98(1), 169-192.

Torres, V. E. \& Celton, D. E. (2009). Discriminación salarial en Argentina entre nativos y paraguayos. Cuadernos geográficos de la Universidad de Granada, 45(2), 263-285.

Verdugo, R. \& N. Verdugo. (1989). The Impact of Surplus Schooling on Earnings. Journal of Human Resources, 24(4), 629-643. doi: 10.2307/145998

Waisgrais, S. (2005). Determinantes de la Sobreeducación de los Jóvenes en el Mercado Laboral Argentino. En Ponencia presentada en el Séptimo Congreso Nacional de Estudios del Trabajo, Retrievedfrom: http:// www.aset.org.ar/congresos/7/10004. pdf 